\title{
Renormalization for Philosophers
}

\author{
J. Butterfield and N. Bouatta \\ Saturday 14 June 2014
}

Forthcoming (slightly abridged) in Metaphysics in Contemporary Physics: a volume of Poznan Studies in Philosophy of Science, eds. T. Bigaj and C. Wüthrich

\begin{abstract}
We have two aims. The main one is to expound the idea of renormalization in quantum field theory, with no technical prerequisites (Sections 2 and 3). Our motivation is that renormalization is undoubtedly one of the great ideas - and great successes - of twentieth-century physics. Also it has strongly influenced, in diverse ways, how physicists conceive of physical theories. So it is of considerable philosophical interest. Second, we will briefly relate renormalization to Ernest Nagel's account of inter-theoretic relations, especially reduction (Section 4).

One theme will be a contrast between two approaches to renormalization. The old approach, which prevailed from ca 1945 to 1970, treated renormalizability as a necessary condition for being an acceptable quantum field theory. On this approach, it is a piece of great good fortune that high energy physicists can formulate renormalizable quantum field theories that are so empirically successful. But the new approach to renormalization (from 1970 onwards) explains why the phenomena we see, at the energies we can access in our particle accelerators, are described by a renormalizable quantum field theory. For whatever non-renormalizable interactions may occur at yet higher energies, they are insignificant at accessible energies. Thus the new approach explains why our best fundamental theories have a feature, viz. renormalizability, which the old approach treated as a selection principle for theories.

That is worth saying since philosophers tend to think of scientific explanation as only explaining an individual event, or perhaps a single law, or at most deducing one theory as a special case of another. Here we see a framework in which there is a space of theories. And this framework is powerful enough to deduce that what seemed "manna from heaven" (that some renormalizable theories are empirically successful) is to be expected: the good fortune is generic.

We also maintain that universality, a concept stressed in renormalization theory, is essentially the familiar philosophical idea of multiple realizability; and that it causes no problems for reductions of a Nagelian kind.
\end{abstract}




\section{Contents}

1 Introduction $\quad 3$

2 Renormalization: the traditional approach 4

2.1 Prospectus: corrections needed . . . . . . . . . . . . . 4

2.2 Renormalizing a coupling constant . . . . . . . . . . . 6

2.2.1 Virtual particles and perturbation theory . . . . . . . . . 7

2.2.2 Energy scales . . . . . . . . . . . . . . . . . . . . . . . 9

2.2 .3 Some Details . . . . . . . . . . . . . . . . 11

2.3 The cut-off introduced . . . . . . . . . . . . . . . . . 12

2.4 Letting the cut-off $d$ go to zero . . . . . . . . . . . . . . . . 13

2.5 The need for extra terms . . . . . . . . . . . . . . . . 16

2.5.1 The physical rationale . . . . . . . . . . . . 16

2.5.2 A refined definition of renormalizability . . . . . . . . . . . 17

2.6 Which theories are renormalizable? . . . . . . . . . . . . 18

2.6.1 Dyson's criterion . . . . . . . . . . . . . . 18

2.6.2 Our good fortune . . . . . . . . . . . . . . . 19

3 The modern approach to renormalization $\quad 20$

3.1 Good fortune explained: non-renormalizable terms dwindle at longer distances . . . . . . . . . . . . . . . . . 20

3.1.1 Decoupling high-energy behaviour . . . . . . . . . . . . 21

3.1.2 Spacetime need not be a continuum . . . . . . . . . . . . 22

3.1.3 Effective theories only? . . . . . . . . . . . . . . . . 23

3.1.4 The renormalization group flow . . . . . . . . . . 25

3.2 Short-distance behaviour: the beta-function and asymptotic freedom . 26

3.3 The perspective from the theory of condensed matter . . . . . . . . . 29

3.3.1 Continuous phase transitions: scale-invariance . . . . . . . . . 30

3.3.2 Critical exponents: the correlation length . . . . . . . . . . . 32

3.3.3 Short distances: a natural lower limit . . . . . . . . . . . . . 35

4 Nagelian reflections $\quad 37$

4.1 Nagel endorsed . . . . . . . . . . . . . . . . . . . . . . . . . . . . . . . . . . . . 38

4.1.1 Deducibility . . . . . . . . . . . . . . . . . 39

4.1.2 Informal constraints on reduction . . . . . . . . . . . . . . . . 40

4.2 Renormalizability deduced at low energies as a family of Nagelian reductions .............................. 42

4.3 Universality is multiple realizability . . . . . . . . . . . . 43

5 References $\quad 44$ 


\section{Introduction}

We have two aims. The main one is to expound the idea of renormalization in quantum field theory, with no technical prerequisites (Sections 2 and 3). Our motivation is that renormalization is undoubtedly one of the great ideas - and great successes of twentieth-century physics. Also it has strongly influenced, in diverse ways, how physicists conceive of physical theories. So it is of considerable philosophical interest. Second, we will briefly relate renormalization to Ernest Nagel's account of inter-theoretic relations, especially reduction (Section 4$){ }^{1}$

One main point will turn on a contrast between two approaches to renormalization. The traditional approach was pioneered by Dyson, Feynman, Schwinger and Tomonaga in 1947-50: they showed how it tamed the infinities occurring in quantum electrodynamics, and also agreed with experiments measuring effects due to vacuum fluctuations in the electromagnetic field - even to several significant figures. After these triumphs of quantum electrodynamics, this approach continued to prevail for two decades. For this paper, the main point is that it treats renormalizability as a necessary condition for being an acceptable quantum field theory. So according to this approach, it is a piece of great good fortune that high energy physicists can formulate renormalizable quantum field theories that are so empirically successful; as they in fact did, after about 1965, for forces other than electromagnetism - the weak and strong forces.

But between 1965 and 1975, another approach to renormalization was established by the work of Wilson, Kadanoff, Fisher etc. (taking inspiration from ideas in statistical mechanics as much as in quantum field theory). This approach explains why the phenomena we see, at the energies we can access in our particle accelerators, are described by a renormalizable quantum field theory. In short, the explanation is: whatever non-renormalizable interactions may occur at yet higher energies, they are insignificant at accessible energies. Thus the modern approach explains why our best fundamental theories have a feature, viz. renormalizability, which the traditional approach treated as a selection principle for theories. (So to continue the metaphor above: one might say that these theories' infinities are not just tamed, but domesticated.)

That point is worth making since philosophers tend to think of scientific explanation as only explaining an individual event, or perhaps a single law, or at most deducing one theory as a special case of, or a good approximation of, another. This last is of course the core idea of Nagel's account of inter-theoretic reduction. The modern approach to renormalization is more ambitious: it explains, indeed deduces, a striking feature (viz. renormalizability) of a whole class of theories. It does this by

\footnotetext{
${ }^{1}$ That Section is brief because one of us (JB) discusses Nagelian themes more fully in a companion paper (2014). From now on, it will be clearest to use 'we' for a contextually indicated community e.g. of physicists, as in 'our best physical theories'; and 'I' for the authors, e.g, in announcing a plan for the paper like 'In Section 2, I will'.
} 
making precise mathematical sense of the ideas of a space of theories, and a flow on the space. It is by analyzing this flow that one deduces that what seemed "manna from heaven" (that some renormalizable theories are empirically successful) is to be expected: the good fortune we have had is generic.

But I will urge that this point is not a problem for Nagel's account of intertheoretic relations. On the contrary: it is a striking example of the power of Nagelian reduction. And I will end with an ancillary point, which also strikes a Nagelian note. I will argue that universality, a concept stressed in renormalization theory, is essentially the familiar philosophical idea of multiple realizability; and I will claim (following Sober, Shapiro and others) that multiple realizability does not cause problems for reductions of a Nagelian kind.

The plan is as follows. I sketch the old and new approaches to renormalization in Sections 2 and 3. ${ }^{2}$ Then in Section 4, I shall maintain that these developments accord with Nagel's doctrines.

\section{Renormalization: the traditional approach}

\subsection{Prospectus: corrections needed}

Consider a classical point-particle acting as the source of a gravitational or electrostatic potential. There is no problem about using the measured force $F$ felt by a test-particle at a given distance $r$ from the source, to calculate the mass or charge (respectively) of the source particle.

Thus in the electrostatic case, for a test-particle of unit charge, the force is given by minus the derivative of the potential energy $V$ with respect to the distance $r$ between the source and the test-particle. In symbols, this is, for a source of charge $e$ (neglecting constants): $F=-\nabla V(r) \sim-\nabla-e / r \equiv-e / r^{2}$. We then invert this equation to calculate that the source's charge is: $e=-F \cdot r^{2}$. (Adding in the constants: $F=\frac{-e}{4 \pi \varepsilon_{0} r^{2}}$, where $\varepsilon_{0}$ is the permittivity of free space (electric constant), implies that $e=-F\left(4 \pi \varepsilon_{0} r^{2}\right)$.)

This straightforward calculation of the source's mass or charge does not work in quantum field theory! There are complicated corrections we must deal with: perhaps unsurprisingly, since it amounts to trying to characterize one aspect of an interacting many-field system in a way that is comparatively simple and independent of the rest of the system. The corrections will depend on the energy and-or momentum with

\footnotetext{
${ }^{2}$ I will give very few references to the technical literature; as perhaps befits a primer. But I recommend: (i) Baez's colloquial introductions (2006, 2009), of which Sections 2 and 3 are an expansion into more academic prose; (ii) Wilson's Scientific American article (1979) and Aitchison (1985)'s introduction to quantum field theory, especially its vacuum, which discusses renormalization in Sections 3.1, 3.4, 3.6, 5.3, 6.1; (iii) Teller (1989) and Hartmann (2001) as philosophical introductions; (iv) Kadanoff's masterly surveys $(2009,2013)$, which emphasize both history and aspects concerning condensed matter-here treated in Section 3.3.
} 
which the test particle approaches the source. A bit more exactly, since of course the test particle and source are equally minuscule: the corrections depend on the energy (or momentum) with which we theoretically describe, or experimentally probe, the system I called the 'source'. We will write $\mu$ for this energy; and the corrections depending on $\mu$ will be centre-stage in both the traditional and the modern approaches to renormalization (this Section and the next).

This Section lays out the traditional approach in four subsections. In Section 2.2, I introduce the idea that the medium or field around the source can affect the observed value of its mass or charge. In quantum field theory, this is often expressed in the jargon of "virtual states" or "virtual particles". Again: it is a matter of the energy-scale $\mu$ at which we describe or probe the source.

Then in Section 2.3, I report that to get finite predictions, quantum field theory needs a regularization scheme. The archetypal scheme is to neglect energies above a certain value $\Lambda$; equivalently, one neglects variations in fields that occur on a spatial scale smaller than some small length $d$. I also adopt length as the fundamental dimension, so that I express regularization as a cut-off length $d$, rather than an energy $\Lambda$.

In Section 2.4, I present the core task of the traditional approach to renormalization. Since the theory assumes spacetime is a continuum, while $d$ is our arbitrary choice, we need to show consistency while letting $d$ tend to 0 . That is: we must find an assignment of intrinsic charges (electric charge, mass etc.: called bare coupling constants), to the sources, as a function of the diminishing $d$, which delivers back the observed value of the charges: i.e. the values we in fact measure at the energy-scale $\mu$ at which we probe the system. These measured values are called the physical coupling constants. If we can do this, we say our theory is renormalizable.

This requirement is weak, or liberal, in two ways. First: we even allow that the assigned intrinsic charge is infinite at the limit $d \rightarrow 0$. (It is this allowance that a bare coupling constants be infinite that makes many -including great physicists like Dirac-uneasy.)

Second (Section 2.5): we allow that we might have to add other terms to our theory (to be precise: to the Lagrangian or Hamiltonian), in order to make a consistent assignment. But we only allow a finite number of such terms: this reflects the fact that our framework of calculation is perturbative.

Then in Section 2.6, I report (the simplest rendition of) Dyson's criterion for when a theory is renormalizable: the dimension (as a power of length) of the bare coupling constant(s) needs to be less than or equal to zero. Finally, I report the happy news that our theories of the electromagnetic, weak and strong forces are in this sense renormalizable. Why we should be so fortunate is a good question: which, as announced in Section 1, I will take up in Section 3. 


\subsection{Renormalizing a coupling constant}

Underlying the details to come, there is a simple idea, which is valid classically and indeed an everyday experience. Imagine a ping pong ball under the surface of the water in a bath. It is buoyant: in terms of the gravitational field, it has a negative mass. So the idea is: the medium in which a system is immersed can alter the parameters associated with the system, even the parameters like an electric or gravitational charge, i.e. its coupling constants.

Agreed, in this example we of course retain the notion that the ball has a positive intrinsic mass, not least because it can be taken out of the water and then will fall under gravity. But three factors make this notion more problematic in fundamental physics, especially in quantum theory.

(i): We cannot take the system out of the field of force, which is all-pervasive, though varying in strength from place to place.

(ii): Even in classical physics, there are important differences between the electromagnetic and gravitational fields: differences that make it wrong, or at least more questionable, to conceptually detach an electric charge from the electromagnetic field, than it is to detach a mass from the gravitational field. In short, the difference is that the gravitational field, but not the electromagnetic field, can be thought of as a mere mathematical device giving the disposition of a test-particle to accelerate: cf. the field $V$ above. On the other hand, the electromagnetic field has energy and momentum, and effects propagate through it at a finite speed: this leads to subtle issues about the self-energy of a classical charged particle. ${ }^{3}$

(iii): In quantum field theory, matter is represented by a quantum field, just as radiation (electromagnetism) is. The matter is represented by a fermionic field; e.g. in quantum electrodynamics, the electron field. And interactions (forces) between matter are represented by a bosonic field; e.g. in quantum electrodynamics, the quantized electromagnetic field, whose excitations are photons. In short: the physical system is an interacting many-field system, so that it makes little sense to conceptually detach one of the fields from the others. All the more so, if we think of our fields as effective, not fundamental: I return to this in Section 3.

In short: we need to take seriously, in our theoretical description as much as our experimental practice, that the system of interest, e.g. an electron (or excitation of the electron field), is immersed in a wider system through which we "access" it. This has two aspects which we need to spell out. The second is more briefly stated, and is fundamental: it will dominate the sequel. But the first sets the stage.

\footnotetext{
${ }^{3}$ For these issues, cf. Zuchowski (2013) and references therein. Broader philosophical aspects of classical fields are discussed by Hesse (1965), McMullin (2002) and Lange (2002).
} 


\subsubsection{Virtual particles and perturbation theory}

First, we need the ideas of: virtual states, also often called virtual particles, which arise in the context of perturbation theory.

In quantum theory, we typically solve a problem by finding the states of definite energy and their corresponding values of energy. These are the energy eigenstates, i.e. eigenstates of the Hamiltonian (energy-function), and their eigenvalues. For once these are known, most of what we might want to know can be calculated. Usually, we cannot exactly calculate the eigenstates: the Hamiltonian is intractable. (The Hamiltonian $H$ is essentially a matrix, and calculating the eigenstates and eigenvalues is a matter of changing bases in the vector space of states so as to render the Hamiltonian matrix diagonal i.e. to make all non-diagonal entries zero.) But often enough, $H$ is "close" to another Hamiltonian, $H_{0}$ say, which is tractable, in that we can calculate $H_{0}$ 's eigenstates. Here, closeness means, roughly, that there is an additional Hamiltonian matrix $H_{i}$ such that $H=H_{0}+\varepsilon H_{i}$ where $\varepsilon$ is a small number. Since $\varepsilon$ is small, $H_{0}$ and $H$ are approximately equal, $H_{0} \approx H$. We can then write the desired eigenstates of $H$ as superpositions (weighted sums of) of the eigenstates of $H_{0}$ (which, recall, we can calculate). Thus $\left|\psi_{a}\right\rangle=\Sigma_{j} c_{j}\left|\psi_{j}^{0}\right\rangle$ : where $a$ labels the real Hamiltonian's eigenvalue (meaning just that $\left.H\left|\psi_{a}\right\rangle=a\left|\psi_{a}\right\rangle\right) ; j$ labels the various eigenvalues of $H_{0}$, whose eigenstates are the $\left|\psi_{j}^{0}\right\rangle$; and our task is to calculate the complex numbers $c_{j}$. It is these eigenstates of $H_{0}$ that are called virtual states or virtual particles.

This jargon is especially used in quantum field theory, where the real Hamiltonian is usually complicated enough to force us to appeal to perturbation theory. This is a general framework for solving intractable problems by treating them as small adjustments to tractable problems: e.g. by adding a term, $\varepsilon V$ say, where $\varepsilon$ is a small number and $V$ a potential function, to the governing equations of the tractable problem. One then tries to calculate the quantity of interest (in our example, one of the $c_{j}$ ) by expressing it as a power series $\Sigma_{n}^{\infty} \alpha^{n} A_{n}$, where $\alpha$ is small, i.e. less than one, so that $\alpha^{n} \rightarrow 0$ as $n \rightarrow \infty$. Here, $\alpha$ may be the original $\varepsilon$, or some related number. The hope is that $\alpha^{n}$ tending to 0 will make the terms $\alpha^{n} A_{n}$ for higher values of $n$ go to 0 . If we are lucky, the first few terms of the series will give us an answer that is accurate enough for our purposes; and if we are very lucky, the series may even converge to the exact answer (i.e. the limit of the successive partial sums $\Sigma_{n}^{N} \alpha^{n} A_{n}$ is finite and is the exact answer). Whether these hopes are realized will of course depend on the $A_{n}$ not growing too quickly.

I should stress immediately that in quantum field theory, the success of this sort of perturbative analysis is mixed. On the one hand, there is astounding success: in some cases, in our best theories, the first few terms of such a series give an answer that is astonishingly accurate. It matches the results of delicate experiments to as much as ten significant figures, i.e. one part in $10^{10}$. That is like correctly predicting the result of measuring the diameter of the USA, to within the width of a human hair! For example, this accuracy is achieved by the prediction in quantum electrodynamics of the magnetic moment of the electron; (Feynman 1985, pp. 6-7, 115-119; Schweber 
1994, p. 206f.; Lautrup and Zinkernagel 1999).

On the other hand, there are serious mathematical problems. It is not just that, in general, the power series expressions we use, even in our best theories, are not known to converge, and are sometimes known not to converge. There are two deeper problems, which I have not yet hinted at.

The first concerns the mathematical definition of the interacting quantum field theory, which our perturbative approach with its various power series is aiming to approximate. Unfortunately, we do not at present have a rigorously defined interacting quantum field theory, for a four-dimensional spacetime. There are such theories for lower spacetime dimensions; and there has been much effort, and much progress, towards the goal. But so far, it remains unattained. One brief way to put the chief difficulty is to say that the central theoretical notion of a quantum field theory, the path integral (also known as: functional integral) - which is what our power series aim to approximate - has at present no rigorous mathematical definition, except in special or simple cases such as there being no interactions. ${ }^{4}$

The second problem is related to the first; it indeed, it is part of it. But the second problem specifically concerns the perturbative approach with its power series, and will be centre-stage in this paper. So it is best stated separately. In short: not only do the power series usually fail to converge; also, the factors $A_{n}$ (in the successive terms $\left.\alpha^{n} A_{n}\right)$ are often infinite. Thus the worry that the $A_{n}$ might 'grow too quickly' for the power series to converge, as I put it above, was a dire under-statement. Nevermind $A_{n}$ being so large for large $n$ that the series might diverge: the problem is really that each term $\alpha^{n} A_{n}$ is infinite! This is quantum field theory's notorious problem of infinities: which, as we will see, is addressed by renormalization. Why the $A_{n}$ are infinite, and how renormalization addresses this by introducing a cut-off and then analysing what happens when the cut-off tends to a limit, will be taken up in Section 2.3 et seq. For the moment, I just confess at the outset that the overall problem of infinities will not be fully solved by renormalization, even by the modern approach (Section 3). The infinities will be tamed, even domesticated: but not completely eliminated. ${ }^{5}$

As an example of treating interactions in quantum theory using perturbation theory, let us consider an electron immersed in, and so interacting with, an electromagnetic field. Here, the electron need not be described by a quantum field; it can be described by elementary quantum mechanics; but we consider the electromagnetic field to be quantized. We take it that we can solve the electron considered alone: that is, we can diagonalize its Hamiltonian $H_{e}$ say - this is essentially what Schrödinger did in 1925. And we take it that we can solve the field considered alone: that is, we can diagonalize its Hamiltonian $H_{f}$ - this is essentially what Dirac did in 1927. But the interaction means that the Hamiltonian of the total system, electron plus field, is not just the (tractable!) sum of $H_{e}$ and $H_{f}$, call it $H_{0}: H_{0}:=H_{e}+H_{f}$. In terms of

\footnotetext{
${ }^{4}$ For a glimpse of these issues, cf. e.g. Jaffe (1999, 2008), Wightman (1999).

${ }^{5}$ But that is perhaps unsurprising since, as I said, this second problem is part of the first. So if it were fully solved, so might be the first problem also.
} 
eigenstates: the energy eigenstates of the total system are not just products of those of the electron and the field; and so the total system's energy eigenvalues are not just sums of the individual eigenvalues.

But happily, the interaction is rather weak. We can write the total Hamiltonian as $H=H_{0}+\varepsilon H_{i}$, where $H_{i}$ represents the interaction and $\varepsilon$ being a small number represents its being weak; and then embark on a perturbative analysis. In particular, we may expand an energy eigenstate in terms of the eigenstates of $H_{0}$, which are each a product of an electron eigenstate and field eigenstate: which latter are states with a definite number of photons (i.e. excitations of the field). So according to the jargon above: these photons will be called 'virtual photons'. ${ }^{6}$ And as I stressed, the theory that treats both the electron and the electromagnetic field as quantum fields which interact with each other, i.e. the theory of quantum electrodynamics, is amazingly empirically accurate. Such an accurate theory is surely getting something right about nature: despite the issues about renormalization, to which we now turn (and some of which, as we shall see later in the paper, are not yet resolved).

\subsubsection{Energy scales}

I said, just before Section 2.2.1, that this second aspect is more briefly stated than the first, but is fundamental and will dominate the sequel. It amplifies the basic point I announced at the start of Section 2: that while in classical physics, there seems no problem about using the measured force felt by a test particle so as to calculate the charge or mass (coupling constant) of the source, this straightforward approach fails in quantum theory - we need to add complicated corrections.

Thus the general, or ideal, classical situation is that our theory says a measured quantity, e.g. a force $F$ on a test particle, is a function of the charge (coupling constant) $g$ of the source: $F=F(g)$; (the function being given by our theory, as in the electrostatic formula, $F=-\nabla-e / r)$. So the task if to measure $F$ and invert this equation to calculate $g$ as a function of $F: g=g(F)$. But in quantum field theory, this approach breaks down: perhaps unsurprisingly, since it amounts to trying to characterize one aspect of an interacting many-field system in a way that is comparatively simple and independent of the rest of the system: recall (iii) at the start of this Section.

Broadly speaking, the corrections depend on the energy and-or momentum with which the test particle approaches the source. A bit more exactly, recognizing that the test particle and source are not conceptually distinguished, since e.g. they might both be electrons: writing $\mu$ for the energy (or momentum) with which we theoretically describe, or experimentally probe, the system, the corrections depend on $\mu$.

So let us write $g(\mu)$ for the physical coupling constant, i.e. the coupling constant that we measure: more exactly, the coupling constant that we calculate from what

\footnotetext{
${ }^{6}$ So NB: 'virtual' does not connote 'illusory', nor 'merely possible', as it does in the jargon of 'virtual work' etc. in classical mechanics - for which cf. Butterfield (2004).
} 
we actually measure, in the manner of $g=g(F)$ above, in the simple electrostatic example. Then the notation registers that $g(\mu)$ is a function of $\mu$. But also: it is a function of the bare coupling constant, $g_{0}$ say, that appears in the theory's fundamental equations (like $e$ ) in the electrostatic example). So we can write $g(\mu) \equiv g\left(\mu, g_{0}\right)$.

Using the details in Section 2.2.1 about virtual states and perturbation theory, we can fill this out a bit. The Hamiltonians of our successful interacting quantum field theories, such as quantum electrodynamics, are indeed intractable, because they include terms (cf. $H_{i}$ in Section 2.2.1) for interactions between the various fields, e.g. the electron field and the electromagnetic field. So we often analyse problems using perturbation theory, and in particular the eigenstates of the free Hamiltonian. Similarly if we formulate our theories using the alternative Lagrangian, rather than Hamiltonian, framework. The Lagrangian function (which is essentially a difference of energy functions) is intractable, because it contains interaction terms; and so again, we turn to perturbation theory.

Usually, for both frameworks and for most problems, perturbation theory yields, as its approximate answer to the problem, a power series in the coupling constant, i.e. $\Sigma_{n} g^{n} A_{n}$; or a power series in some closely related number. Note that getting a power series in the coupling constant is unsurprising, given Section 2.2.1's remark that one often gets a power series in the parameter $\varepsilon$, which in the interaction term $H_{i}=\varepsilon V$, looks like a coupling constant. Cf. Section 2.2.3 for some details.

Besides, as one would guess for a power series in the coupling constant: increasing the exponent $n$ from term to term corresponds to considering corrections which take account of successively more interactions between the fields concerned. So the other factor in each term, viz. $A_{n}$, encodes many ways that there can be $n$ such interactions. In particular as we will see in Sections 2.2.3 and 2.3: it allows for these interactions occurring in various places in space and time (by $A_{n}$ being an integral over the various places, typically over all of space between two times), and at various energies and momenta (by integrating over energies and momenta).

Here we connect with the idea of Feynman diagrams. We think of the interactions as occurring between particles, i.e. between excitations of the fields, and we represent the idea that the interaction occurs at a point $x$ in spacetime by drawing lines coming from the past and reaching to the point, for the incoming particles, and lines from the point towards the future, for the outgoing particles. So the point becomes a vertex of a diagram of lines, and the diagram corresponding to the term $g^{n} A_{n}$ will in general have $n$ vertices. Furthermore, since our perturbative analysis essentially involves calculating eigenstates of the full interacting Hamiltonian by expanding them in states of the free Hamiltonian: in the detailed calculation of the contribution $g^{n} A_{n}$ made by a single diagram, the internal lines represent propagations of virtual states/particles. ${ }^{7}$

\footnotetext{
${ }^{7}$ For some details, cf. e.g. Aitchison (1985: Sections 3.4-3.6, 5.3) and Feynman (1985, especially pp. 115-122). They both discuss the phenomenon of vacuum polarization, and so screening: the intuitive idea is that $g(\mu)$ will be greater at high energies because the test particle penetrates further past the cloud of virtual particles that surround the source, and so "feels" a higher coupling constant.
} 


\subsubsection{Some Details}

By way of filling out Sections 2.2.1 and 2.2.2, here are some details of elementary timedependent perturbation theory. This will be enough to suggest how in a quantum field theory we often work with a power series in the coupling constant $g$; and to connect a term of such a series with a Feynman diagram. (NB: nothing in this Subsection is needed later, so it can be skipped!)

So suppose again that the full Hamiltonian $H$ is $H_{0}+H_{i} \equiv H_{0}+\varepsilon V$, where the parameter $\varepsilon$ is small, and we expand in powers of $\varepsilon$. We define the interaction picture by

$$
|\bar{\psi}(t)\rangle:=\exp \left(i H_{0} t / \hbar\right)|\psi(t)\rangle \quad ; \quad \bar{V}(t)=\exp \left(i H_{0} t / \hbar\right) V \exp \left(-i H_{0} t / \hbar\right) .
$$

so that the equation of motion of $|\bar{\psi}(t)\rangle$ is

$$
i \hbar \frac{d}{d t}|\bar{\psi}(t)\rangle=\varepsilon \bar{V}(t)|\bar{\psi}(t)\rangle .
$$

We assume a solution is a power series in $\varepsilon$ :

$$
|\bar{\psi}(t)\rangle=\Sigma_{n=0}^{\infty} \varepsilon^{n}\left|\bar{\psi}_{n}(t)\right\rangle
$$

with $\left|\bar{\psi}_{n}(0)\right\rangle=0$ if $n>0$, since at $t=0$ the state $|\bar{\psi}\rangle$ is the initial state $\left|\psi_{0}\right\rangle$ independent of $\varepsilon$.

Substituting the power series into the equation of motion and equating powers of $n$ gives a sequence of equations. The $n$th equation is:

$$
i \hbar \frac{d}{d t}\left|\bar{\psi}_{n}(t)\right\rangle=\bar{V}(t)\left|\bar{\psi}_{n-1}(t)\right\rangle
$$

These can be solved successively to give:

$$
\left|\bar{\psi}_{n}(t)\right\rangle=\frac{1}{(i \hbar)^{n}} \int_{0}^{t} d t_{n} \int_{0}^{t_{n}} d t_{n-1} \cdots \int_{0}^{t_{2}} d t_{1} \bar{V}\left(t_{n}\right) \cdots \bar{V}\left(t_{1}\right)\left|\psi_{0}\right\rangle
$$

So

$$
|\bar{\psi}(t)\rangle=\Sigma_{n=0}\left(\frac{\varepsilon}{i \hbar}\right)^{n} \int_{0}^{t} d t_{n} \int_{0}^{t_{n}} d t_{n-1} \cdots \int_{0}^{t_{2}} d t_{1} \bar{V}\left(t_{n}\right) \cdots \bar{V}\left(t_{1}\right)\left|\psi_{0}\right\rangle .
$$

Carrying this over to quantum field theory: in short, the factors $\bar{V}\left(t_{i}\right)$ are replaced by a product of field operators $\phi$, at a spacetime point $x$, representing an interaction between the fields at that point. So a factor replacing $\bar{V}\left(t_{i}\right)$ looks like $\hat{\phi}_{1}\left(x_{i}\right) \hat{\phi}_{2}\left(x_{i}\right) \ldots \hat{\phi}_{k}\left(x_{i}\right)$ to represent $k$ different fields interacting at the spacetime point

In Section 3.2, we will see the opposite phenomenon, anti-screening or asymptotic freedom, where $g(\mu)$ is a decreasing function of energy. 
$x_{i}$. So increasing the exponent $n$ from term to term in a power series in the coupling constant, $\Sigma_{n} g^{n} A_{n}$, amounts to taking account of successively more interactions between the fields concerned. And in each term of the power series, the other factor, viz. $A_{n}$, encodes many ways that there can be $n$ such interactions. In particular, it allows for these interactions:

(i) occurring in various places in space and time, by $A_{n}$ being a $n$-fold multiple integral over the spacetime points $\left(x_{i}, i=1, \ldots, n\right)$ : typically, an integral over all of space between two times; and

(ii) occurring at various energies and momenta, by $A_{n}$ integrating over various possible energies and momenta of the interacting particles, especially the intermediate virtual particles.

As discussed above, the ideas in (i) and (ii) lead to Feynman diagrams.

\subsection{The cut-off introduced}

In the closing remark of Section 2.2.3, that $A_{n}$ integrates over various possible energies and momenta, lurks the notorious problem of quantum field theory's infinities: the second problem of Section 2.2.1 which, as announced there, is addressed by renormalization.

Typically, $A_{n}$ is a (multiple) integral) over energy or momentum $k$, extending from some value, say $k_{0}$ (maybe zero), upto infinity of a function that increases at least as fast as a positive power of $k$, say $k^{a}$. So $A_{n}$ looks like $\int_{k_{0}}^{\infty} d k k^{a}$. If $a>0$, this integral is infinite; as $k \rightarrow \infty$, so does $k^{a}$, for positive $a$, making the integral infinite.

So to get a finite answer from our formulas, we impose a cut-off: we replace the upper limit in the integral, $\infty$, by a suitably high energy or momentum, written $\Lambda$. (There are other 'less crude' ways to secure a finite answer-called regularizing the integrals - but I will only consider cut-offs.)

I have introduced the cut-off as an energy $\Lambda$. But in quantum theory, energy is like the reciprocal of distance; in the jargon, 'an inverse distance': energy $\sim 1 /$ distance. (And so distance is like an inverse energy.) This is due to the fundamental equations, due to de Broglie, relating 'particle' and 'wave' properties: viz. that momentum $p$ is inversely proportional to wavelength $\lambda$ with proportionality constant $h$. That is: $p=h / \lambda$. (NB: $\lambda$ and $\Lambda$ are very different: an unfortunate notational coincidence, but a widespread one ...) Wavelength is the number of units of length per complete cycle of the wave. So writing $k$ for the reciprocal, called wave-number, i.e. the number of wave-cycles per unit-length, we have: $p=h k$. So high momenta (and high energies) correspond to high wave-number, which means short wavelengths and high frequencies.

So the cut-off energy $\Lambda$ corresponds, in terms of distance, to a cut-off at a small

distance $d$. That is: imposing the cut-off, i.e. requiring by fiat that $\int_{\Lambda}^{\infty} d k \ldots \equiv 0$ means ignoring contributions to the integrand that vary on a distance shorter than $d$. In other words: to get finite answers, we are declaring that the theory claims there 
are no fields varying on scales less than $d$. At least: any such fields do not contribute to the specific process we are calculating. Or at least: the theory claims this, unless and until we consider the limit $d \rightarrow 0$ - which we will do in Section 2.4.

So returning to the notation of Section 2.2.2: the physical coupling constant, $g(\mu)$, is a function, not only of the bare coupling constant $g_{0}$ and of $\mu$ itself of course, but also of the cut off $d$. Thus:

$$
g(\mu) \equiv g\left(\mu, g_{0}, d\right)
$$

So we can now state our task, introduced at the start of Section 2, more precisely. We are to measure $g(\mu)$ (better: to calculate it from what we really measure, like the force $F$ in the simple electrostatics example) and then invert eq. 2.7, i.e. write $g_{0}=g_{0}(g(\mu), d)$, so as to calculate which value of the bare constant would give the observed $g(\mu)$, at the given $d$. This task is the core idea of the traditional approach to renormalization.

It is sometimes convenient, for the sake of uniformity, to express all dimensions in terms of length. Section 2.6.1 will give more details. But for the moment, just note that we can trade in the energy-scale $\mu$ for an inverse length, say $\mu \sim 1 / L$ where $L$ is a length. NB: $L$ is not the cut-off $d$ ! We can think intuitively that $L$ is experimental, and $d$ is theoretical. That is: $L$ is our choice about how microscopically to describe or probe - to peer into - the system. On the other hand, $d$ is a (generally much smaller) length below which we are taking our theory to say there are no contributions. So we re-express the physical coupling constant $g(\mu)$ as a function of $L$ : we will use the same letter $g$ for this function, so that we write $g(L) \equiv g(\mu)$. Thus eq. 2.7 becomes:

$$
g(L) \equiv g\left(L, g_{0}, d\right)
$$

In the next two sections, I turn to two further aspects of the task just described: of inverting eq. 2.8 and assigning values to $g_{0}$ that give the observed $g(\mu)$. These aspects concern:

(i) letting $d \rightarrow 0$;

(ii) needing to allow for some extra terms in the equations: which will return us to the analogy of the ping pong ball, at the start of Section 2.2.

\subsection{Letting the cut-off $d$ go to zero}

Broadly speaking, the exact value of the cut-off $d$ is up to us. Agreed: for some of the troublesome infinities - some of the infinite terms $A_{n}$ in perturbative analyses of some problems - the physics of the problem will suggests a range of values of $d$ that are sensible to take. That is: the physics suggests that no phenomena on scales much smaller than $d$ will contribute to the process we are analysing. One such example, very famous for its role in the establishment of quantum electrodynamics, is the Lamb shift, where the electron's Compton wavelength seems a natural lower limit to $d$; cf. Aitchison (1985, Section 3.5-3.6), Schweber (1994, pp. 223-247). 
But of course, we would like the theory and its predictions to be independent of any human choice. So generally speaking, it is natural to take $d$ smaller and smaller, at fixed $\mu$; and to consider how $g_{0}$ varies as a function of $d$, while preserving the observed $g(\mu)$.

More precisely: if we believe that:

(a) spacetime is continuum, and

(b) our theory holds good in principle at arbitrarily short lengths, then we surely also believe that at fixed $\mu$ (or at least at some, maybe theoretically judicious or appropriate, $\mu$ : such as the observed $\mu$ ), $g_{0}$ goes to a limit: that is:

$$
\text { there exists } \lim _{d \rightarrow 0 ;(g(\mu) \text { fixed at observed value) }} g_{0} .
$$

We will later treat the issues that (i) since we can vary $\mu$, there are various observed values $g(\mu)$, and therefore (ii) whether we should require eq. 2.9 for all the observed values $g(\mu)$. We will also treat letting $\mu$ go beyond the observed range, even letting it go to infinity, although we cannot measure $g(\mu)$ above some technological (financial!) maximum value of $\mu$.

If the limit in eq. 2.9 exists and is finite, i.e. $\in \mathbb{R}$, we say: the theory is finite. As the label suggests: in the face of the troublesome infinities, such a conclusion would be a relief. But I should stress some limitations of this conclusion. There are three obvious general ones; (cf. the references in footnote 4):

(i): this limit being finite does not imply that any of the power series which our perturbative analysis provides for specific physical problems converges;

(ii): even if such a series, for a problem at a given value (or range) of $\mu$, does converge, this does not settle the behaviour of the corresponding series at other $\mu$; and that behaviour might be bad - in particular, arbitrarily high $\mu$ might produce a troublesome infinity;

(iii): even if all goes well in a perturbative analysis, i.e. the various series converge for the ranges of parameters for which we might hope, there remains a gap between a perturbative analysis and the original physics problem or problems.

But even with these prospective limitations, some successful quantum field theories are not finite. The paradigm case is QED. For QED, the limit in eq. 2.9 is infinite. That is: for arbitrarily high cut-offs, the bare charge $g_{0}$ is arbitrarily high (and remains so for yet higher cut-offs). Mathematically, this is like elementary calculus where we say that some function $f(x)$ tends to infinity as $x$ tends to infinity, e.g. $\lim _{x \rightarrow \infty} \sqrt{ } x=\infty$. But of course this last is 'just' the infinity of pure mathematics. But here we face (assuming (a) and (b) above) a physically real infinity viz. as the value of the bare coupling constant.

The consensus, on the traditional approach to renormalization, is that this physically real infinity is acceptable. After all: since by construction we do not measure 
(nor calculate from measurements) the bare constant, we do not need to allow an 'Infinity' reading on our apparatus' dials. To reflect this consensus, the adjective 'renormalizable', with its honorific connotations, is used. That is: If the limit in eq. 2.9 exists, albeit perhaps being $\pm \infty$, we say the theory is renormalizable. So in particular: QED is renormalizable in this sense, though not finite. (This definition of 'renormalizable' will be filled out in the next two subsections.)

But I should add that despite this consensus, most physicists would admit to some discomfort that the bare constant should be infinite in the continuum theory that, according to (a) and (b), we are thinking of as fundamental. Thus great physicists like Dirac have been very uncomfortable (cf. the citations in Cao (1997, pp. 203-207)); and Feynman himself calls renormalization 'a dippy process' and 'hocus-pocus' (1985, p. 128); (Teller (1989) is a philosophical discussion).

Besides, this discomfort does not depend on believing exactly (a) and (b) above. Suppose that instead we merely believe the corresponding claims of possibility:

(a') spacetime might be a continuum, and

(b') our theory should be a consistent description (of the interactions in question) at arbitrarily short lengths.

In short: we merely believe that our theory, formulated with a continuum spacetime as background, is a "way the world could be". Then we surely are committed to believing that at fixed $\mu$ (or at least some, maybe theoretically judicious or appropriate, $\mu), g_{0}$ goes to a limit. And again, it is uncomfortable that this limit is infinity. Although this yields, not an actual physical infinity, but only a possible physical infinity: surely a philosopher should be uncomfortable at such a possibility. (Section 3.1.3 will return to this, suggesting a way to ease the discomfort.)

But despite this discomfort, the fact remains that after facing the troublesome infinities, it is obviously a great intellectual relief to find one's theory to be renormalizable, even if not finite. It means we can succeed in our task: namely, to consistently assign bare constants (albeit perhaps infinite ones) so as to recover the observed physical coupling - and do so independently of the cut-off $d$ we adopt so as to make integrals finite.

This relief prompts the idea that even if one does not explicitly endorse (a) and (b) (or perhaps, even ( $\mathrm{a}^{\prime}$ ) and (b')), one should adopt renormalizability as a reasonable criterion for selecting theories. Thus the idea is: a good theory of whatever interactions, should make sense, albeit perhaps with an infinite bare coupling constant, when formulated with a continuum spacetime as background. This is indeed how renormalizability was regarded in the traditional approach to renormalization, which reigned ca. 1950 to 1970: acceptable theories should be remormalizable. 


\subsection{The need for extra terms}

The main issue in trying to write down a renormalizable theory is that we may need to add (to the Lagrangian or Hamiltonian function) one or more terms to represent extra interaction(s) between the fields, even though we believe the bare coupling constant for the extra interaction(s) are zero. I first describe (i) the physical rationale for this; and then (ii) the refinement it prompts in the definition of renormalizability, and thereby in what the task of formulating a renormalizable theory involves.

\subsubsection{The physical rationale}

Thus suppose we think the bare coupling constant of some interaction is zero. That is, we think that in our fundamental theory, a certain interaction - which would typically be represented by some product of field operators - does not happen. Then we will be tempted to have no term representing this interaction in our theory (as a summand in our Lagrangian or Hamiltonian). For whatever the form, say $\mathcal{F}$, of the interaction (i.e. of the product of operators), we will think we can leave the term out of all equations. For if $g=0$ then the term $g . \mathcal{F}$ equals zero, and surely contributes nothing to any equations.

But this might be a mistake! Despite the zero bare coupling, the interaction might have a non-zero physical coupling constant at some scale $\mu$; i.e. $g(\mu) \neq 0$. Indeed, this situation can arise not only for:

(a): the strength of a certain interaction between given fields; but also for

(b): the mass or charge of a given field, or as people say: the mass or charge of a given particle (treated as an excitation of a field).

In case (b), we would be tempted to omit as pointless terms for all possible interactions of the given field (particle) that depend on that mass or charge, since the terms are apparently zero, and so surely contribute nothing to any equations. But this might be a mistake: the physical coupling constant may be non-zero at some scale $\mu$. In such a case, we say: 'the field (or particle) acquires a mass/charge at the scale $\mu$ '.

The analogy of the ping pong ball, mentioned at the start of Section 2.2, may help. There, the fact that it falls in a vacuum (or air) but is buoyant in wateri.e. exhibits a positive gravitational mass in vacuum and air, but a negative one in water - illustrated the general idea that the coupling constants associated to a system can be altered by the medium in which the system is immersed. But now imagine the ping pong ball is so light as to be massless in air, so that in air, it does not fall under gravity but floats, weightless; yet when immersed in water, it 'acquires a mass' (in fact a negative one, since it moves upwards, opposite to the force of gravity). Thus a

\footnotetext{
${ }^{8}$ Intuitively, case (b) seems more problematic than case (a): for the mass or charge of a given field seems more "intrinsic" to it than is participation in a certain interaction with other fields, and our habituation to mass and charge in classical physics makes us think such properties are "given" prior to any interactions, rather than acquired from them.
} 
system with $g_{0}=0$ might have at some scale $\mu$ a non-zero physical coupling constant, $g(\mu) \neq 0$, which you could measure; (better: calculate from actual measurements).

So faced with this situation, whether case (a) or case (b): we should of course include the corresponding term or terms in our fundamental equations. For recall that our basic task is to find values of the bare constants that (at the given $\mu$ and $d$ ) imply the measured values of $g(\mu)$. Here it will help to generalize the notation slightly to reflect the fact that there are of course several, even many, coupling constants to consider; as well as several, even many, possible interactions (terms in the Lagrangian or Hamiltonian that are typically products of operators). So suppose that there are in all $N$ physical coupling constants, $g_{1}(\mu), g_{2}(\mu), \ldots g_{N}(\mu)$, occurring in the various terms/interactions in our theory. Then we cannot expect to have them implied by less than $N$ bare constants, even if we think some of the bare constants are zero. After all, to fit $N$ numbers, we expect to need $N$ numbers.

\subsubsection{A refined definition of renormalizability}

So we now envisage a number $N$ of different coupling constants; and we recognize that we might have to allow extra terms for interactions, in particular those whose bare couplings are zero (at least in the limit of greatest interest, viz. $d \rightarrow 0$ ). This suggests a more sophisticated, indeed more flexible, task than I stated before (cf. after eq. 2.7 in Section 2.3). The task is still to assign bare constants so as to recover the measured physical constants, and in particular so as to secure the limit in eq 2.9. But now we are allowed to add (if need be) extra terms: terms which can be judiciously selected by us the theorist.

It seems reasonable to say that such extra terms are legitimate hypotheses to add to our initial theory (our initial collection of terms), provided that all the terms taken together, together with the limiting values of the bare constants given by eq 2.9, imply the measured values of the various $g(\mu)$. After all: we have at least 'saved the phenomena' with our theory formulated on a spacetime continuum, albeit perhaps with the cost of judiciously selected extra terms. And this seems legitimate, even if (as conceded in Section 2.4) some of the limiting values of the bare constants are $\infty$. Indeed: this seems legitimate, even if some of the limiting values of the bare constants in the new additional terms selected by us theorists are $\infty$ - even though we originally motivated such terms by the case where the limiting value of the additional bare constant is zero. ${ }^{9}$

In any case, whether or not you would call it 'reasonable': this is the consensus, on the traditional approach to renormalization, under one proviso. Namely, that there should be only a finite number of extra terms. The idea is: our theory should not qual-

\footnotetext{
${ }^{9} \mathrm{By}$ focussing on renormalization, I have of course set aside the other requirements that the theory must satisfy, if we are to talk of 'legitimate hypotheses' and 'saving the phenomena'. To include those requirements, I should of course add something like: 'and provided that the theory is otherwise empirically successful'.
} 
ify as 'saving the phenomena' if we have to make infinitely many such augmentations to it. That is: a theory which secures the limit in eq 2.9, using either no extra terms, or only a finite number of them, is given the honorific adjective: renormalizable.

\subsection{Which theories are renormalizable?}

I end this Section's review of the traditional approach to renormalization by very briefly reporting: (i) the criterion for when a theory is renormalizable, and (ii) that our empirically successful quantum field theories satisfy this criterion. The good fortune in (ii) will prompt the question: why should we be so lucky? Section 3 will take up this question (using the criterion in (i)).

\subsubsection{Dyson's criterion}

Suppose we focus, not on a whole theory as given by a Lagrangian or Hamiltonian, i.e. by a sum of terms for the various sorts of energy of the various fields and their various interactions; but on a single such term. If you like, we imagine a theory so simple as to contain only one term. It turns out that the criterion for this theory, i.e. term, to be renormalizable, can be simply stated.

To do so, we should first express all dimensions in terms of length. We saw in Section 2.3 that, thanks to de Broglie's relation $p=h / \lambda$, we can trade in a cut-off in energy $\Lambda$ for a distance $d$, and similarly the energy-scale $\mu$ for a distance $L$; with higher energies corresponding to shorter distances, so that e.g. $\mu \sim 1 / L$. (Recall that $L$ is not the cut-off $d$.) But it turns out that we can go much further: not only energy but all quantities can be expressed as powers of length, by invoking the idea (due to Planck) that two fundamental constants, such as the speed of light $c$ and Planck's constant $h$, can be declared to be dimensionless, instead of (as usual) thinking of them as having respectively dimensions 'length divided by time' and 'length times momentum'. The idea is that after making this declaration, we 'back-calculate' what dimension some familiar quantity such as an electric charge must have, so that our equations come out as dimensionally consistent. In this sort of way, a quantity turns out to have as its dimension some power of length: it has dimension length ${ }^{D}$. Here, the power (also called: exponent) $D$ can be positive or negative. For example, $L^{-1} \equiv 1 / L$, so that with $h$ declared dimensionless, de Broglie's relation $p=h / \lambda$ implies that momentum has dimension length ${ }^{-1}$. For brevity, this is often shortened by dropping mention of length, so that we say: 'momentum has dimension -1 '.

We can now state the criterion for a term (in the Lagrangian) to be renormalizable. It turns out that this is so iff: the bare coupling constant which the term contains has dimensions of length ${ }^{D}$, with $D \leq 0$. This is called Dyson's criterion, or the power-counting criterion.

More precisely: suppose that the bare coupling constant $g_{0}$ has dimensions of length ${ }^{D}$. Then the corresponding physical coupling constant $g(\mu) \equiv g(L)$ will scale 
roughly like $L^{-D}$. That is:

$$
g(L) / g_{0} \sim(L / d)^{-D}
$$

Thus if $D>0$, the exponent on the right-hand side will be negative; so when $L$ is very small, i.e. much smaller than $d$, the right hand side is very large. That is: the physical coupling constant will be large compared with the bare one. That is a sign of bad behaviour at small distances $L$, i.e. high energies. At least, it is bad in the sense that the large coupling constant will prevent our treating the interaction represented by the term as a small perturbation. Thus it is perhaps unsurprising that such a term is non-renormalizable in the sense sketched informally at the end of Section 2.5. ${ }^{10}$

Eq. 2.10 will also be important in the modern approach to renormalization. To anticipate a little: Section 3.1 will examine the case $D>0$, i.e. non-renormalizability, for large distances; $L$ and Section 3.2 will examine the "happy" case of $D \leq 0$, even of small $L$.

\subsubsection{Our good fortune}

So much for the general ideas. How do the quantum field theories we "believe in", or "take seriously" fare? That is: are the theories which are our best descriptions of the electromagnetic, weak and strong forces, renormalizable in the sense just discussed?

In short, they fare very well. For first: quantum electrodynamics (QED) is renormalizable in this Dyson sense. As to the other two forces: we have since the 1970s had:

(i) a unified theory of the electromagnetic and weak forces (the electro-weak theory of Weinberg and Salam; also called '(quantum) flavour-dynamics' (QFD); and

(ii) a theory of the strong force (quantum chromodynamics, QCD). (Like QED, these theories are so far defined only perturbatively; but unlike QED, they each use a non-abelian gauge group: QFD uses $S U(2) \times U(1)$ and QCD uses $S U(3)$.) And indeed: both of these are renormalizable.

So all three - QED, QFD and QCD - are renormalizable. But we should recall that all three theories are defined only perturbatively: recall that we do not have a rigorously defined interacting quantum field theory in four spacetime dimensions (Section 2.2.1), and that even a finite theory is defined only perturbatively and may harbour divergent series (Section 2.4). Because of these limitations, a more modest jargon is appropriate. So a qualifying adverb is often added to the honorific 'renormalizable'. Namely, we say these three theories are perturbatively/superficially renormalizable.

It seems a piece of great good fortune that our best theory of some force of nature be renormalizable (even perturbatively): let alone our theories of three such forces. At least, it is a relief after (a) having to admit that we can so far only define the theory perturbatively, and (b) having to face, from Section 2.1 onwards, complicated

\footnotetext{
${ }^{10}$ This bad behaviour is not to say that a non-renormalizable theory is mathematically inconsistent: e.g. the Gross-Neveu model is non-renormalizable.
} 
corrections: a relief that the theory can in the above sense 'save the phenomena', even if it is not finite in Section 2.4's sense.

But we will now see that according to the modern approach to renormalization, this great good fortune is not so surprising. In a certain sense, renormalizability is generic at the low-ish energy scales we can access - cf. the next Section.

\section{The modern approach to renormalization}

The key initial idea of this approach, initiated in the mid-1960s by the work of Wilson, Fisher, Kadanoff and others (with important earlier work by e.g. Stueckelberg, GellMann and Low) is that instead of being concerned with good limiting behaviour as the cut-off $d \rightarrow 0$, we instead focus on how $g(\mu)$ varies with $\mu$. In terms of the ping pong ball analogy at the start of Section 2.2, and Section 2.2.2's discussion of energy scales: we now focus, not on regularizing integrals with a cut-off $d$, but on how the parameters of a system, e.g. the mass of a ping pong ball, depend on the energy or momentum scale at which we describe it.

Indeed, if we envisage a number of coupling constants, say $N$ for $N$ possible interactions, then the "vector" of coupling constants $\left(g_{1}(\mu), \ldots, g_{N}(\mu)\right)$ represents a point in an $N$-dimensional space; and as $\mu$ varies, this point flows through the space. And accordingly: if we envisage a theory as given by a Lagrangian (or Hamiltonian) which is a sum of terms representing different possible interactions, then this space is a space of theories. Jargon: we say the coupling constants run, and the flow is called the renormalization group flow.

As we shall see, this simple idea leads to a powerful framework. I shall first (Section 3.1) report how it explains why a theory (like QED) that concerns phenomena at the low (or low-ish!) energy scales that we can access, is renormalizable. That is: it explains why the good fortune noted in Section 2.6.2 is generic. Then in Section 3.2, I discuss high-energy, i.e. short-distance, behaviour. Finally, I discuss insights about the renormalization group that come from thinking about statistical mechanics (Section 3.3). All three Subsections will introduce some jargon, indeed "buzz-words", such as (respectively): fixed points, asymptotic freedom and universality.

\subsection{Good fortune explained: non-renormalizable terms dwin- dle at longer distances}

To explain "our good fortune" in the sense introduced in Section 2.6.2 is to explain why a theory about phenomena at the low, or moderate, energy scales that we can access should be renormalizable. There are of course various philosophical controversies about explanation. But I take it to be uncontroversial that one very satisfying way to explain this would be to show: not merely that some given theory is renormalizable; but that any theory, or more modestly, any of a large and-or generic class 
of theories, is renormalizable. To the extent that the class of theories is indeed large and-or generic, such an argument would demonstrate that our good fortune was "to be expected". (Admittedly, such an explanation, whether for a single theory, or for a class of them, will have to make some other assumptions about the theory or theories: a point I will stress in Section 3.1.1. So it is only relative to those assumptions that the good fortune is explained, and to be expected.)

This is indeed what the modern approach to renormalization gives us, with its idea of a space of theories, on which there is a flow given by varying the energyscale $\mu$. More precisely and modestly, but also more practically: I admit that this approach does not show that any of a large and-or generic class of theories has, at the comparatively low energies and large length-scales we can access, literally no non-renormalizable terms. Rather, the approach shows that for any such theory"with whatever high-energy behaviour, e.g. non-renormalizable terms, you like" - the non-renormalizable terms dwindle into insignificance as energies become lower and length-scales larger. That is, in Section 2's notation: the physical coupling constant for non-renormalizable terms shrinks. For such terms: as $\mu \rightarrow 0$ (i.e. $L \rightarrow \infty$ ), $g(\mu) \equiv g(L) \rightarrow 0$.

Indeed, this explanation is already clear from Section 2.6.1's discussion of eq. 2.10: which, to repeat it, was:

$$
g(L) / g_{0} \sim(L / d)^{-D}
$$

In Section 2.6.1, we focussed on the case where $L$ is very small, so that a nonrenormalizable term's positive exponent (in the dimension of length) makes for a large physical coupling constant. But just look at the other side of the same coin. When $L$ is large (much larger than the cut-off $d$ ), and $D>0$ (i.e. the term in question is non-renormalizable), then the right hand side of eq. 3.1 is very small. That is: the physical coupling constant is very small. So at large distances, the nonrenormalizable interaction is weak: "you will not see it".

There are four main points I should make about this explanation, before discussing short-distance behaviour (Section 3.2). The first point is about how non-trivial the explanans, i.e. eq. 3.1, is. The second point is about the explanation not depending on spacetime being a continuum. This will prompt the third point, about effective theories. (The second and third points, in Sections 3.1.2 and 3.1.3, correspond to Sections 5.2.1 and 5.2.2 of the companion paper (2014).) The fourth point will somewhat generalize the discussion, from a physical not philosophical viewpoint; and will introduce some more jargon.

\subsubsection{Decoupling high-energy behaviour}

That at large distances, a non-renormalizable interaction is weak follows immediately from eq. 3.1. But that does not make it obvious! A good deal of theory needs to be assumed in order to deduce eq. 3.1. After all, there is of course no a priori guarantee 
that interactions that are strong at short distances should be weak at long distances. To show this "decoupling" of high-energy behaviour from the low-energy behaviour was a major achievement of Wilson, the other authors mentioned, and indeed many other physicists, e.g. Symanzik (1973), Applequist and Carazzone (1975). I will not go into details, but just make three general remarks.

(i): It can be shown under very general conditions, even within the confines of a perturbative analysis.

(ii): Looking ahead: Section 3.1.3 will mention Weinberg's perspective, based on a result roughly to the effect that, even without assuming the framework of quantum field theory ab initio, any relativistic quantum theory's description of physics at low enough energies must look like the description given by a quantum field theory.

(iii): Again, looking ahead: Section 3.2 will say a bit more about how the limiting high-energy behaviour of $g(\mu)$ is encoded in a function, the beta-function, which can be calculated perturbatively.

\subsubsection{Spacetime need not be a continuum}

Notice that this explanation does not depend on our theory (with all its terms, including non-renormalizable ones) being true, or even approximately true, at arbitrarily short distances. It only needs to be approximately true at suitable intermediate distances. More precisely: it only needs to secure eq. 3.1 holding for any nonrenormalizable interaction at a range of scales which is wide enough to include $L$ being sufficiently larger than the cut-off $d$, so that with the given positive dimension $D$ of the bare coupling constant, the left hand side of eq. 3.1 is small enough that we will not see the interaction.

We can put the same point in more physical terms, and in terms of energies. Maybe at very high energies, spacetime does not behave like a continuum. But provided the theory is "true enough" at some high, maybe even inaccessible, energies in the sense that it validates eq. 3.1, then we can deduce that at much lower, in particular accessible, energies, "we see only renormalizable interactions". That is: our theory's predictions have significant contributions only from renormalizable interactions.

Note incidentally that this independence of spacetime being a continuum is much stronger than we saw before, in the shift in Section 2.4, from assuming (a) and (b) to assuming (a') and (b'): roughly, the shift from assuming that our theory described physics in a continuous spacetime to assuming merely that it might do so. In the present argument, we could be agnostic about whether, or even deny that, our theory could describe physics in a continuous spacetime. All we need is that it is approximately true at suitable intermediate distances, as just specified.

Here we meet a widespread jargon. A theory that is taken to be approximately true in a given regime (of energy and-or length, and-or some other parameters) is called effective. The adjective is used especially when the theory is known or believed to be only approximately true; say, because it is derived using certain approximating 
and-or idealizing assumptions (assumptions which go beyond merely specifying the regime, i.e. range of parameters, concerned).

So we can sum up the above explanation of what I called 'our good fortune' by saying: from studying the renormalization group flow, we deduce (subject to the theoretical assumptions gestured at in Section 3.1.1) that effective low-energy theories are renormalizable. The idea of effective theories leads in to the next point.

\subsubsection{Effective theories only?}

I ended Section 2.4 by reporting that on the traditional approach, renormalizability functioned as a criterion for selecting theories. But the explanation at the start of Section 3.1 undermines this stance. For it says that, although non-renormalizable terms induce bad behaviour, i.e. a large coupling, at short distances, this bad behavour is invisible at the larger distances we can access. So why worry? That is: why not countenance non-renormalizable terms, at least for inaccessibly high energies?

Of course, the words 'worry' and 'countenance' are vague. What you are inclined to worry about, and correspondingly what you are willing to countenance, will depend on your background attitudes to quantum field theory: for example, on how confident you are about using it at high energies, and about accepting results obtained from a heuristic formalism, rather than by rigorous mathematical proofs. So there are bound to be several possible positions. Here I will briefly develop one position, often called the effective field theory programme (or: approach). It is based, not so much on confidence about the two topics above, as on an opportunistic or instrumentalist attitude to being unconfident about them. (In Section 3.2, I will describe a less opportunistic or instrumentalist attitude, based on results showing some quantum field theories' good behaviour at arbitrarily short distances.)

There are of course two main factors that prompt a cautious or sceptical attitude towards the framework of quantum field theory.

(1): One is just that interacting quantum field theories (in four spacetime dimensions) are at present mathematically ill-defined. Recall that this was the first of the two serious mathematical problems listed in Section 2.2.1.

(2): The other factor is the expectation that at sufficiently high energies, the framework breaks down, to be replaced by a theory or theories using a different framework. This break-down might occur only at the vast energies associated with quantum gravity: the replacement theory being perhaps a version of string theory, or some other current contender for a theory of quantum gravity. Or the break-down might occur at intermediate energies, energies far higher than we can (and probably: ever will) access, but well below those of quantum gravity: there are proposals for new frameworks at these energies, such as non-commutative geometry.

Either or both of these factors prompt one to be cautious about drawing from quantum field theory conclusions about ontology. Or rather: conclusions about the ontology of phenomena at very high energies, or very short distances. But these 
factors should not suspend all discussion of ontology in the light of physics, or even in the light of quantum field theory; for three reasons.

(a): Whatever the phenomena at very high energies turn out to be, whatever the theoretical framework for describing them, and whatever ontology that framework suggests, we have every reason to expect that the facts at those energies determine (in philosophical jargon: subvene) the facts at the lower energies we can access.

(b): And given the great success of quantum field theory, we have every reason to expect that the facts at those very high energies imply a quantum field theoretic description at the lower, accessible, energies.

(c): Besides, whoever said that ontology concerns only "the supervenience basis", i.e. the putative set or level of facts that determine (subvene) all other facts? That is: there is plenty of scope for ontological discussion of supervening ("higher level") facts and theories: in particular, there is scope for for ontological discussion of quantum field theory.

But these factors also suggest that even below the energy scale at which the entire framework of quantum field theory breaks down, there may, for all we know, not be any single quantum field theory which is more fundamental than the others, in the sense that each of them is derived from it by assuming extra conditions that specify the derived theory's regime (of energies and types of interaction considered etc.). That is: as the energy scale gets higher and higher (while remaining below the scale at which the entire framework of quantum field theory breaks down), physics might be described by a succession of quantum field theories, each of which accurately describes the phenomena at a certain range of energies, but becomes inaccurate above that range. And when it becomes inaccurate, it may also become even more badly behaved, mathematically.

This scenario is often called the tower of effective field theories. But the phrase can be misleading, for two complementary reasons.

(i): First, as I mentioned when defining 'effective', at the end of Section 3.1.2: the adjective is often used when the theory is known or believed to be only approximately true, because it is derived using approximating and-or idealizing assumptions. But in this scenario, the theories in the envisaged tower are not required to be derivable from some other theory: in particular, one with greater credentials, or warrant, for being taken as exactly true ('fundamental') because it also covers higher energies. Rather, each theory is simply accurate in its energy range, and inaccurate beyond it.

(ii): Second: the word 'tower' suggests an infinite tower. But as I noted in (1) above, there are good reasons (concerning quantum gravity if nothing else) to think that at some energy, the entire framework of quantum field theory breaks down. So as a programme or approach for quantum field theory, the effective field theory programme can, and should, admit that the tower is probably finite.

But setting aside misleading connotations: the main point is that this scenario gets some support from this Section's explanation of "our good fortune", viz. that any non-renormalizable interactions (terms), though they would be important at higher 
energies, will make a dwindling contribution to all processes, as the energy scale is reduced. For this explanation implies that we cannot get evidence about which nonrenormalizable interactions, if any, operate at inaccessibly high energies. Whatever they are - and whatever the bad short-distance behaviour they suffer (cf. the end of Section 2.6.1) — we will not see them. So why worry about non-renormalizable interactions (terms)? And for all we know, or could ever know, the scenario of the tower holds good: there is no fundamental quantum field theory, and various such interactions operate at various inaccessibly high energies.

There is a further, and substantial, point to make. So far, my exposition of the effective field theory scenario has had the spirit of epistemic modesty: "for all we know". A true and worthy sentiment, if a bit dull. But Weinberg has developed a stronger and more positive perspective on the matter. It provides an answer to the question why physics at accessible energies should be described by a quantum field theory at all, even if the framework breaks down higher up, e.g. because of gravity. And this answer yields the narrative strategy for his magisterial exposition of quantum field theory (1995; cf. pp. xx-xxi, 1-2, 31-38; 1999 pp. 242-247). In short, there is the following result; (admittedly, with 'result' understood by the standards of heuristic quantum field theory, not pure mathematics). Any quantum theory that at low enough energies is Lorentz-invariant and satisfies one other main assumption, called 'cluster decomposition' (which is plausible, since it has the flavour of a locality assumption), must at low enough energies be a quantum field theory (1999, p. 246).

So much by way of sketching the effective field theory programme. We can sum it up as urging that, regardless of how and why quantum field theory might break down at very high energies (as it presumably does, thanks to gravity, if nothing else): we have no reason in theory, nor experimental data, to deny the scenario of the towera succession of theories, each accurately describing physics in its energy range, and inaccurate beyond it.

As I said at the start of this Subsection, this suggests a rather opportunistic or instrumentalist attitude to quantum field theories. I will return to this briefly at the start of Section 4. Meanwhile, in Section 3.2, I will describe how results showing some quantum field theories' good behaviour at arbitrarily high energies foster a less opportunistic or instrumentalist attitude. More precisely: the results suggest that there are good prospects that these theories can be rigorously defined (pace (1) above).

\subsubsection{The renormalization group flow}

So far, my talk of the renormalization group flow has been restricted in three ways; which we need to overcome. The most important is that we need to consider, not just the flow as energy $\mu$ decreases (length $L$ increases), but also the flow in the other direction: as $\mu$ increases ( $L$ decreases). This needs a separate subsection: Section 3.2. Here I overcome two smaller restrictions: this will also introduce more jargon.

(a): A flow can have a fixed point, i.e. a point that is not moved by the flow: 
think of sources and sinks in elementary discussions of fluid flow. In our context (the renormalization group flow), this would mean a set of physical coupling constants $\left(g_{1}(\mu), \ldots, g_{N}(\mu)\right)$ that is unchanged as $\mu$ decreases further (as the length-scale increases further). Jargon: the behaviour of the system is scale-invariant: "you see the same behaviour/theory/physical coupling constants, at many different length-scales". This can indeed happen; and we will see a vivid physical reason for this, related to statistical mechanics, in Section 3.3. Such a point is called an infra-red fixed point. Here, 'infra-red' is used on analogy with light: infra-red light has a longer wavelength, lower frequency and lower energy, than visible light.

(b): So far, we have had in mind one trajectory, maybe leading to a fixed point. But many trajectories might lead to the same fixed point; or at least enter and remain in the same small region of the space. If so, then the 'vectors' $\left(g_{1}(\mu), \ldots, g_{N}(\mu)\right)$ at diverse early points on a pair of such trajectories representing dissimilar theories lead, as $\mu$ decreases, to the same fixed point, or at least to the same small region, and so to similar theories. That is: when you probe at low energies/long distances, "you see the same physical coupling constants/behaviour/theory". Jargon: This is called universality. And the set of 'vectors' that eventually lead, as $\mu$ decreases, to the given fixed point is called, on analogy with elementary discussions of fluid flow, the point's basin of attraction. But note that universality should really be called 'commonality' or 'similarity': for there can be different fixed points, each with their own basin of attraction. But jargon aside: Section 3.3 will give more physical details about universality, and Section 4.3 will assess whether it is different from the familiar philosophical idea of multiple realizability.

Finally, we can summarize this Subsection's main point, that non-renormalizable interactions dwindle at large length-scales, by combining the jargon we have just introduced with the previous jargon that a free theory is a theory with no interactions. Namely: the infra-red fixed points of theories all of whose terms are nonrenormalizable are free theories.

\subsection{Short-distance behaviour: the beta-function and asymp- totic freedom}

Instead of considering the flow as energy $\mu$ decreases (length $L$ increases), we can of course consider flowing in the other direction: as $\mu$ increases ( $L$ decreases). Again, the jargon is borrowed from light: we can consider the flow towards the ultra-violet. Looking again at eq. 3.1 (which is eq. 2.10), we see that it is terms/interactions for which $D<0$ for which the physical coupling constant goes to zero as $L \rightarrow 0$; since for these terms, the physical coupling constant scales like $L$ to a positive power.

Of course, the coupling constant being zero means the interaction is not "seen" (cf. Section 2.5.1). The behaviour we see is that of the free, i.e. non-interacting, theory. This is called asymptotic freedom. And as in (a) in Section 3.1.4, this free theory may be fixed, i.e. not moved, by the flow. If so, it is an ultra-violet fixed point. 
On the other hand, if $D=0$, then according to eq. 3.1, the physical coupling constant scales like $L$ to the zeroth power; that is, it is constant. More precisely: we need to study the range under which eq. 3.1, or some more precise equation, is valid, and what happens to the physical coupling constant(s) beyond that range.

So these cases, $D<0$ and $D=0$, are very different; accordingly, there is jargon to distinguish them. Recall that in Section 2.6.1, we called a term for which $D \leq 0$ 'renormalizable'. But we now distinguish the two cases. If $D<0$ (the "happy case"), we say the theory is super-renormalizable. If $D=0$, we say the theory is ("merely") renormalizable. But if in this latter case, a more subtle analysis shows that the coupling constant goes to zero as $L \rightarrow 0$, we will still say the theory is asymptotically free. That is: this buzz-word is not reserved for super-renormalizable theories.

We can summarize, using the jargon we have just introduced, like we did at the end of Section 3.1.4. Namely: asymptotically free theories, in particular superrenormalizable theories, have a free theory as an ultra-violet fixed point.

Note that the idea of a ultra-violet fixed point is more general than asymptotic freedom, in that the renormalization group flow could have a non-free theory as an ultra-violet fixed point. The various possibilities for what happens to $g(\mu)$ as $\mu$ tends to infinity are often described in terms of the beta-function, which is defined by

$$
\beta(g):=\frac{d g}{d \ln \mu} \equiv \mu \frac{d g}{d \mu} .
$$

Here $\ln \mu$ is the logarithm of $\mu$. So the $\beta$-function is the rate of increase of $g$ with respect to a logarithmically damped measure $\ln \mu$ of the energy: since logarithm rises very slowly, it is in effect an amplified rate of increase of $g$ with respect energyamplified by multiplying by the energy itself.

So as $\mu$ tends to infinity, there are three possibilities for $g(\mu)$ having a finite limit, i.e. an ultra-violet fixed point. Since there is a fixed point, all will involve $\lim _{\mu \rightarrow \infty} \beta=0$. But $g$ might have zero as its limit (as discussed: asymptotic freedom). Or $g$ might have some non-zero value $g_{\star}$ as its limit. This is called asymptotic safety. Or $g$ might be a constant, $g_{\star}$ say, independent of $\mu$; so that $g$ does not, colloquially speaking, tend to a limit - it is already there. This situation occurs in theories which are conformally invariant. ${ }^{11}$

To summarize, the three possibilities for $g(\mu)$ having a finite limit at short distances are:

(a): asymptotic freedom: $\lim _{\mu \rightarrow \infty} \beta=0 ; \lim _{\mu \rightarrow \infty} g=0$;

(b): asymptotic safety: $\lim _{\mu \rightarrow \infty} \beta=0 ; \lim _{\mu \rightarrow \infty} g=g_{\star} \neq 0$.

(c): conformal invariance: $\beta \equiv 0$ i.e. $g$ is constant, independent of $\mu$. Compare Figure 1; where the ultra-violet fixed point is the dot. In Fig. 1(a), as $\mu$

\footnotetext{
${ }^{11}$ This means, roughly speaking, that the theory is symmetric under any change of scale (a dilation). This extra symmetry makes conformally invariant theories easier to study in various ways (especially if spacetime has only one spatial dimension); and thus important to us, even though they do not describe the known forces.
} 
grows the negative $\beta$ drives $g$ down to 0 . In Fig. 1(b), as $\mu$ grows, a positive $\beta$ drives $g$ up towards the fixed point, while a negative $\beta$ drives it down. Finally in Fig. 1(c), $g$ is constant independent of $\mu .^{12}$

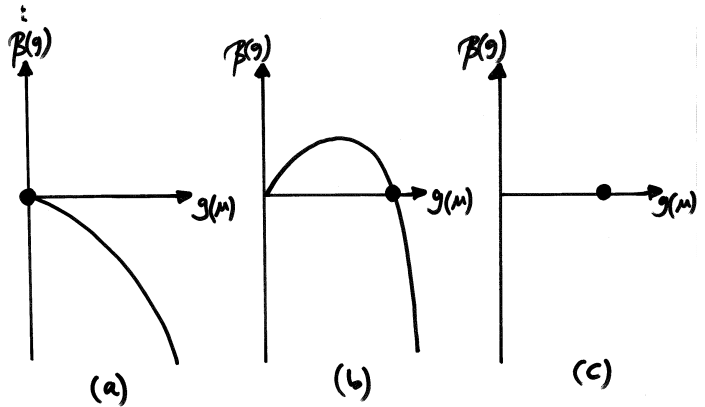

Figure 1: UV fixed points

So much for the general ideas. Let us ask, as we did in Section 2.6.2: How do the quantum field theories which are our best descriptions of the electromagnetic, weak and strong forces, get classified? There, we reported that all three (i.e. QED, QFD and QCD) are renormalizable - in that Section's inclusive sense, that $D \leq 0$ : i.e. $D<0$ or $D=0$. More exactly, they are perturbatively renormalizable, since as emphasized there, the theories have not yet been rigorously defined.

Now that we distinguish the two cases, $D<0$ vs. $D=0$, there is: bad news and good news - indeed, there are two pieces of each. First, the bad news: (that is, in addition to the prevailing lack of rigour). First: None of the theories is superrenormalizable. They are "merely" renormalizable; so we need a more subtle analysis of their short-distance behaviour. Because the three theories are only defined perturbatively, it is hard to make such an analysis. But there is every reason to believe that for QED, there is more bad news; (this is the second piece of bad news). Namely: QED's is badly behaved at short distances. That is: in QED, as $L$ decreases, the coupling constant, i.e. the charge of the electron, at first looks constant-but it then grows and grows. There is good reason to think it tends to infinity, as $L \rightarrow 0$.

On the other hand: for QCD, the corresponding analysis yields good news - good short-distance behaviour. That is: There is every reason to believe that QCD is asymptotically free. So at very short distances, quarks do not feel each other's weak or strong force. ${ }^{13}$ Besides, there may be some good news about gravity. In this paper,

\footnotetext{
${ }^{12}$ My thanks to Nazim Bouatta for Figure 1, and for teaching me most of this Section. Of course, one can give a more fine-grained classification than Figure 1's (a)-(c): cf. e.g. the options in Weinberg (1995a, Section 18.3, p. 130f.).

${ }^{13}$ Wilczek's Nobel lecture (2005) is a beautiful and masterly introduction to asymptotic freedom, especially in QCD. QFD is, unfortunately, not asymptotically free. Its high energy behaviour is complicated: for details cf. e.g. Horejsi (1996), Moffat (2010); thanks to Nic Teh for these references.
} 
I have of course ignored gravity, apart from saying in Section 3.1.3 that people expect quantum gravity to force a breakdown of quantum field theory. One main reason for that is the fact that the quantum field theory obtained by quantizing general relativity is not renormalizable: and thereby, on Section 2's traditional approach, not acceptable. But there is evidence that this theory is asymptotically safe, i.e. that the physical coupling constant has a finite limit at high energies, case (b) above; (Weinberg 1979, Section 3, p. 798f).

This good news prompts a broader point, which was foreshadowed at the end of Section 3.1.3's discussion of effective theories. Namely, asymptotic freedom suggests these theories can be rigorously defined. This is not to suggest that success is over the next hill: if attainable, it is some way off-but asymptotic freedom gives us grounds for optimism. ${ }^{14}$ If so, this would count against the effective field theory vision, that (regardless of gravity) there is a succession of theories, each accurately describing physics in its energy range, but inaccurate beyond it.

\subsection{The perspective from the theory of condensed matter}

No account of the modern approach to renormalization, however brief, would be complete without some discussion of the role therein of ideas from the theory of condensed matter. ('Condensed matter' is short for 'liquid or solid'.) Ideas from this field have been invaluable. To convey something of these insights, I shall make just three main points: that continuous phase transitions correspond to infra-red fixed points of the renormalization group flow (Section 3.3.1); that renormalization group methods enable us to calculate correctly the critical exponents of such transitions (Section 3.3.2); and finally, that in a condensed matter system, there is a natural lower limit to the cut-off $d$ and length $L$ (Section 3.3.3). ${ }^{15}$

At the outset, I should clarify my usage. I will contrast 'theory of condensed matter', with 'quantum field theory', understood as I have done hitherto in this paper: viz. as describing high energy physics, especially the fundamental forceselectromagnetism, the weak force and the strong force. But I stress that the mathematics of quantum field theory is used endemically to describe condensed matter. For example, one often describes a solid or liquid with a quantum field (say: energy or momentum, or electric field): this amounts to assigning a quantum operator to each point of space or spacetime - thus abstracting away from the atomic constitution of matter. I will briefly return to this in Section 3.3.3.

\footnotetext{
${ }^{14}$ Besides, we can show that if a theory rigorously exists, then its asymptotic freedom can be ascertained perturbatively: so there is no threat of future success undermining our present grounds for optimism. For this pleasant surprise, cf. Gross (1999, p. 571).

${ }^{15}$ Condensed matter is, fortunately, more familiar than quantum fields. Among many approachable references for this material, let me pick out just Kadanoff's masterly surveys $(2009,2013)$, Batterman (2010), and Menon and Callender (2013).
} 


\subsubsection{Continuous phase transitions: scale-invariance}

In both classical and quantum physics, understanding condensed matter is usually harder than understanding gases, since the mutual proximity of the atoms makes for stronger interactions, and so for problems that are harder to solve (cf. Section 2.2.1's discussion of intractable Hamiltonians). So it is little wonder that most of the early successes of statistical mechanics - which is the core theory for understanding condensed matter - concerned gases. But the last half-century has seen great strides in understanding liquids and solids, in both classical and quantum physics.

Most relevant to us is the topic of phase transitions. These are transitions between macroscopically distinguishable states (also called: phases), such as the liquid, solid or gaseous states themselves. So melting and freezing, boiling and condensing, are all phase transitions; but so is the change in the direction of magnetization of some material, under the influence of a changing magnetic field. Here, I will consider only a special kind of phase transition, called continuous or second-order, or a critical point. The idea is that in such a phase transition, the material "looks the same" at whichever length scale you examine it (scale-invariance): this phenomenon of scale-invariance does not occur in the more usual first-order phase transitions.

Thus consider water boiling, i.e. liquid water being heated and becoming steam. Usually - for example in a kettle - the phase transition is first-order: there is a body of water that is almost entirely liquid but for a few bubbles of steam rising in it, and a body of steam (and air), but for a few droplets of liquid water. If we think of the density as the quantity whose varying values encode the difference between the phases, liquid and solid, there is no scale-invariance. For the two regions where liquid and gas predominate are separated by the bubbling surface; and besides, on a smaller scale, the bubbles of steam in the liquid (and the droplets of water in the gas) have some characteristic mean size (and variance).

But there is a special temperature and pressure, called the critical point, at which the distinction between liquid water and steam breaks down, and there is scaleinvariance. That is: scrutinizing a volume of liquid water, we see that it contains bubbles of steam of widely varying sizes in roughly equal proportions; and scrutinizing such a bubble, we see that it contains yet smaller droplets of water, which are themselves of widely varying sizes in roughly equal proportions; and if we scrutinize one of those droplets, we find it contains yet smaller bubbles ... and so on, through many orders of magnitude of size, until we reach molecular dimensions, where, of course, the alternation between the phases breaks down. ${ }^{16}$

Thus the critical point involves a "tower of self-similarity", where zooming in to see finer detail presents the same picture as we saw at larger length-scales. More precisely: it presents the same sort of picture, in a statistical sense. That is: the exact position,

\footnotetext{
${ }^{16}$ This critical point for water happens at a temperature of 374 degrees Celsius $(647=374+273$ degrees Kelvin). The water-steam mixture takes on a cloudy appearance so that images are blurred; and thus the phenomenon is called 'critical opalescence'. As we will see in Section 3.3.2, it also happens for other liquid-gas mixtures.
} 
size and other properties of the bubbles of steam, at any level, is of course a matter of myriadly complicated happenstance. But the statistical properties of the bubbles at different levels match, in the sense that: if we specify the degree of magnification (zooming in: the change of length-scale) between two levels, there is a corresponding re-scaling of the bubbles' other quantities, such as expected size, density etc., which maps the means and variances of bubbles' quantities at the first level to those at the second. In short: there is statistical self-similarity, under changes of length-scale, through many orders of magnitude, until we reach molecular dimensions.

Many other phase transitions, in many other materials, can occur in this curious, statistically self-similar, way in which the idea of a boundary between the phases breaks down; (unsurprisingly, this requires special external conditions, like temperatures, pressures etc.). For example, it can happen in a magnet. The analogue to the alternation between bubbles of steam and droplets of liquid water is the alternation between the magnetization in two different spatial directions, for example "up" and "down". At the critical point (requiring a special temperature, the Curie temperature), a region of the magnet with magnetization predominantly up turns out to contain "islands" whose magnetization is predominantly down, but each such island contains contain islands whose magnetization is predominantly up ... and so on.

We can already see how the idea of a critical point connects with several of the notions in Section 3.1, especially the renormalization group flow, infra-red fixed points and universality (Section 3.1.4). Zooming out our description of a system to longer distances corresponds to flowing to lower energies (decreasing $\mu$ ) in a quantum field theory. Scale-invariance means that the description does not change as we zoom out further. So such a description corresponds to an infra-red fixed point of the renormalization group flow.

Furthermore, we can strengthen my phrase 'corresponds to' to is, if we make the notion of 'description' of the condensed matter system more precise as the set of physical coupling constants that occur in the Hamiltonian that describes the system at the distance-scale concerned. (Similarly, with Lagrangian instead of Hamiltonian; but for short, I will just say 'Hamiltonian'.)

That is: we can set up the idea of a space of theories of condensed matter systems, and a flow on this space, just as we did at the start of Section 3. Given a postulated microscopic Hamiltonian describing the atomic or molecular interactions, the zooming out process is then a matter of successively coarse-graining the description, i.e. defining collective variables, to give an effective Hamiltonian. The standard example, viz. the Ising model, is very intuitive. The system is a regular array of sites (in one or more dimensions: a line or a lattice); with each of which is associated a variable taking one of two values, +1 or -1 . The microscopic Hamiltonian encodes the idea that equal values for neighbouring spins are "preferred" (i.e. have lower energy). One coarse-grains by taking a majority vote of the values in a block of, say, ten spins; thus defining a new array, with ten times fewer sites, described by a new effective Hamiltonian. One then defines a flow on the space of Hamiltonians 
by iterating this coarse-graining; for details, cf. the maestro's exposition (Kadanoff 2013, Section 6.4, p. 170-172). Thus a Hamiltonian that is unchanged by zooming out, i.e. is scale-invariant, is precisely an infra-red fixed point of this flow.

Finally, note that the notion of universality carries over directly to the context of condensed matter physics. It means that two disparate physical systems, with very different microscopic Hamiltonians, can be described at long length-scales in the same way. (This of course sounds exactly like philosophers' notion of multiple realizability: cf. Section 4.3.) Indeed: if the two microscopic Hamiltonians flow to the same infra-red fixed point, then the two systems are described at their respective critical points, by the very same Hamiltonian. (And similarly, for descriptions in the neighbourhood of the critical point: the descriptions of the two systems are close but not identical.) Having the same Hamiltonian can make the two systems have exactly equal values for corresponding quantities. This quantitative equality, despite very disparate microscopic constitutions, can be very striking - as we will see in the next Subsection.

\subsubsection{Critical exponents: the correlation length}

One aspect of the quantitative behaviour of materials at and near a continuous phase transition, is the fact that the values of various quantities are given by power-laws, i.e. by the value of some other quantity, raised to some power. More specifically: critical points occur only at a specific temperature, the critical temperature $T_{c}$; and near the critical point, the value of some quantity, $v(Q)$ say, is given by a power of the difference between the actual temperature $T$ and $T_{c}$, or by a power of some similar quantity such as the ratio of this difference to $T_{c}$ :

$$
v(Q) \sim\left|T-T_{c}\right|^{p} \quad \text { or } \quad v(Q) \sim\left|\frac{T-T_{c}}{T_{c}}\right|^{p}
$$

where $p$ is the power.

It is worth giving some examples of how the same power law (same $p$ ) can govern the critical points of very disparate systems. This will show how striking universality can be: which will be relevant to the philosophical discussion in Section 4.3. Besides, since the renormalization group framework can correctly predict these power laws for countless such systems, it will show the amazing success of the framework. My examples will be drawn from condensed matter, not quantum field theory, since this will be technically less demanding: water and steam are familiar, while quarks and gluons are not. But I stress that this Section's themes - phase transitions, critical points, universality and the renormalization group successfully predicting the power laws - nowadays also form a large subject within quantum field theories like QCD; (e.g. Kogut and Stephanov 2004). But note: the details in the rest of this Subsection are not needed in my closing philosophical discussion (Section 4), and so can be skipped; nor will Section 4 need these themes in the form they take within quantum field theories. 
In eq. 3.3, $Q$ might be a quantity whose value is zero at the critical point (so that $p$ is positive). For example, $Q$ might be:

(i) the difference $\rho_{\text {liquid }}-\rho_{\text {gas }}$ between the densities of liquid water and of steam; or

(ii) the average magnetization $m$ of a piece of iron.

Or $Q$ might be a quantity that diverges (i.e. goes to infinity) at the critical point (so that $p$ is negative). For example, $Q$ might be

(i') the isothermal compressibility $\kappa_{T}$ of water: a measure of how compressible it is, i.e. how the volume changes as a function of pressure, at fixed temperature $T$ (formally: $\kappa_{T}=-\partial V /\left.\partial p\right|_{T}$ ); or

(ii') the magnetic susceptibility of iron: a measure of how magnetizable it is, i.e. how its magnetization changes as a function of an external applied magnetic field $B$, at fixed temperature $T$; (formally: $\chi_{T}=-\partial m /\left.\partial B\right|_{T}$ ).

In these four examples, it is obvious enough that $(i)$, the difference $\rho_{\text {liquid }}-\rho_{\text {gas }}$, vanishes at the critical point. For as I sketched in Section 3.3.1, each body of liquid water contains much gas, which contains much liquid, and so on ... and vice versa. A similar argument can be made that (ii) vanishes, and (i') and (ii') diverge, at their respective critical points. But we do not need the details of why that is so, in order to make universality vivid.

All I need is to report the striking, indeed amazing, fact that the two members of each pair ((i) and (ii), (i') and (ii')) obey the same law. That is: (i) and (ii) obey the same power-law, namely with $p \approx 0.35$. In fact this $p$ is written as $\beta$. So we have

$$
\rho_{\text {liquid }}-\rho_{\text {gas }} \sim\left|T-T_{c}\right|^{\beta} \text { and } m \sim\left|T-T_{c}\right|^{\beta} ; \quad \text { with } \quad \beta \approx 0.35 \text {. }
$$

Furthermore, this power-law, with almost the same value of $\beta$, describes corresponding phase transitions in many other systems. Here is an example like (i): as one reduces the temperature of a sample of liquid helium below $2 \mathrm{~K}$ (two degrees above absolute zero, i.e. -271 degrees Celsius) another phase of helium (a superfluid phase: so-called because it flows without viscosity) condenses out, rather like liquid water condensing out of steam. This transition has a power law like eq. 3.4 with $\beta \approx 0.34$. Another example like (ii) is the magnetization of nickel: here, $\beta \approx 0.36$.

Similarly, (i') and (ii') obey the same power-law, with nearly equal values of their exponent $p$ : namely $p \sim-1.2$. By convention, this $p$ is written as $-\gamma$, so that $\gamma$ is positive. So we have

$$
\kappa_{T} \sim\left|T-T_{c}\right|^{-\gamma} \text { and } \quad \chi_{T} \sim\left|T-T_{c}\right|^{-\gamma} ; \quad \text { with } \gamma \approx 1.2
$$

Again, this power-law, with almost the same value of $\gamma$, describes corresponding phase transitions in many other systems. Here is an example like (i'): a mixture of two organic fluids (viz. trimethylpentane and nitroethane) has a critical point rather like that of water-and-steam: the compressibility $\kappa_{T}$ obeys a power law like eq. 3.5, with $\gamma \approx 1.24$. And for helium, i.e. our previous example like (i): the compressibility 
has $\gamma \approx 1.33$. Another example like (ii') is the magnetic susceptibility of nickel: here, $\gamma \approx 1.33$.

$\beta$ and $\gamma$ are called critical exponents. By convention, critical exponents are defined to be positive; so that if the quantity concerned diverges at the critical point, the exponent/index in the power-law is minus the critical exponent. There are several others. All of them encode some power-law behaviour of quantities at or near a critical point: and each of them takes very nearly the same value for critical points occurring in materials and processes that are microscopically very disparate. So again, we see a striking universality of systems' quantitative (albeit arcane!) behaviour.

I need not go into details, except to describe how two critical exponents, written $\eta$ and $\nu$, encode the power-law behaviour near the critical point - not of straightforwardly macroscopic quantities like the density, compressibility etc. so far mentionedbut of measures of the microscopic correlations. Sketching this will bring us back to Section 3.3.1's central idea of scale-invariance.

One of course expects correlations between the states at different locations in a material to decrease, as one considers more widely separated locations. Indeed, this is so: and at or near critical points, the decrease is often given by a power of the separation. Thus suppose we define as our measure of correlation, the expectation (probabilistic average) of the product of local variables (often called 'order parameters') at the two locations, separated by distance $r$. This is often written $G(r) .{ }^{17}$ At the critical temperature $T_{c}, G(r)$ obeys a power law, for $r$ large compared with inter-molecular distances. Namely:

$$
G(r) \sim 1 / r^{d-2+\eta}
$$

where (i) $d$ is the spatial dimension of the material (usually 3; but e.g. 2 for a monoatomic or monomolecular layer, or a theoretical model of such), and (ii) $\eta$ is typically between 0 and 0.1 . (In my examples, it is: (a) for the mixture of two organic liquids and for helium, 0.02; (b) for iron, 0.07; (c) for nickel, 0.04.)

Near $T_{c}, G(r)$ obeys, not a simple power law in $r$, but an exponential decrease:

$$
G(r) \sim \exp (-r / \xi) ;
$$

which defines the length $r=\xi$ over which $G(r)$ decreases by about $66 \%$ ( $e \approx 2.7$ so that $\left.\exp (-\xi / \xi) \equiv e^{-1} \approx 1 / 3\right)$. This means that $\phi$ fluctuates in correlated blocks of various sizes up to a length $\xi$, but blocks with a side-length much bigger than $\xi$ are very rare.

It is here that we see again the scale-invariance of the critical point-and so its being an infra-red fixed point of the renormalization group flow. For as $T_{c}$ is

\footnotetext{
${ }^{17}$ It is usually defined in terms of local variables $\phi$ by: (i) assuming spatial homogeneity so that any two locations a distance $r$ apart yield the same expectation, and one may as well choose the origin $\mathbf{0}$ and some location $\mathbf{r}$ which is $r$ units away, so that one would write $G(r)=\langle\phi(\mathbf{0}) \cdot \phi(\mathbf{r})\rangle$, where \langle\rangle indicates expectation; and (ii) subtracting away any background constant correlation in $\phi$, the better to study fluctuations, so that one writes $G(r):=\langle\phi(\mathbf{0}) \cdot \phi(\mathbf{r})\rangle-|\langle\phi\rangle|^{2}$.
} 
approached from above or from below, $\xi$ grows without limit. Indeed, its behaviour is described by (yet another!) power law: with a negative exponent so as to capture the divergence, as with the $-\gamma$ for (i') and (ii') above. That is: near $T_{c}$ (i.e. $\left|T-T_{c}\right| / T_{c}<<$ 1), we have

$$
\xi \sim\left|T-T_{c}\right|^{-\nu}
$$

where $\nu$ is typically about $2 / 3$. Again, the approximate value $2 / 3$ covers a whole class of phenomena: for our four examples of the critical points for the two organic liquids, helium, iron and nickel, the range of $\nu$ is from 0.62 (the liquids) to 0.69 (iron). ${ }^{18}$

Finally, a note of caution. My praise of the modern approach to renormalization, with its successful calculation of critical exponents (and, needless to say: so much else!), might give the impression that only with the renormalization group did physics cotton on to the broad idea of coarse-graining a microscopic description to give a macroscopic one, or of iterating a scheme for coarse-graining, so as to understand scale-invariant phenomena, and in particular to calculate critical exponents.

That is not so. Before the renormalization group analyses were developed, various approaches to understanding these phenomena had considerable, albeit partial, success. That is of course hardly surprising, since the ideas of coarse-graining, and iterating a scheme for coarse-graining, are so natural. But it is worth emphasizing: not just so as to honour the previous work, but also to avoid philosophers getting the false impression that only with the renormalization group did physics pick up on the idea of multiple realizability. In short: the renormalization group is of course a scientific triumph, and a stimulus to philosophical relection - but that does not imply that it harbours entirely novel morals for philosophy. Section 4 will expand on this irenic theme. ${ }^{19}$

\subsubsection{Short distances: a natural lower limit}

This Section has stressed the analogy between the renormalization group framework for quantum field theory and for condensed matter. But I should end by noting two main differences; the second will be more important. (Of course, there are also many differences of detail.)

First: I have spoken impartially of the renormalization group flow going (i) towards long distances, the infra-red (Sections 3.1 and 3.3.1), and (ii) towards short distances,

\footnotetext{
${ }^{18}$ This discussion, especially the data cited, is based on Binney et al. (1992, pp. 7-8, 18-20, 22).

${ }^{19}$ Two broad previous approaches are mean field theory and Landau theory. For a glimpse of what these are and their predictive limitations, in the context of condensed matter, cf. e.g. Kadanoff (2009, Sections 2,3; 2013, Sections 1.2.4-4, pp. 147-164) and Binney et al. (1992, pp. 22, 176-177, 183-184). In particular, mean field theory implies values for the critical exponents I have discussed, as follows: $\beta=0.5, \gamma=1, \eta=0, \nu=0.5$. As to the false impression that within physics only the renormalization group describes multiple realizability: I think this impression is fostered by some philosophers' praise of the renormalization group; e.g. Batterman (2002, pp. 37-44), Morrison (2012, p. 156 , p.160 (both paragraph 2)).
} 
the ultra-violet (Section 3.2). But broadly speaking, a flow towards the infra-red is often defined by (iterated) coarse-graining. And since coarse-graining involves a loss of microscopic information, such a flow is in general many-one, i.e. irreversible: this underpinned Section 3.3.1's idea of two disparate microscopic Hamiltonians flowing to the same infra-red fixed point. So there is no well-defined inverse flow towards the ultra-violet. (And in the jargon of group theory, one should thus say 'renormalization semi-group', not 'renormalization group'; but the latter name has stuck.) This point is not meant to suggest there is a problem, or even a puzzle, about such irreversible flows. The exact definition of the flow is up to the theorist, and of course varies from theory to theory, and according to the theorist's purposes. Thus the flow towards the infra-red is not always defined by coarse-graining (loss of information); so in some cases it is indeed reversible, and there is a well-defined flow to the ultra-violet. ${ }^{20}$

The second difference is about the ultra-violet regime. Namely: quantum field theory lacks, but condensed matter has, a natural lower limit to the lengths $d$ and $L$. In more detail: quantum field theories, being field theories, assume a spacetime continuum. Although I pointed out that we can make sense of such theories without spacetime being a continuum, namely as describing physics at low enough energies, large enough distances (Sections 3.1.2 and 3.1.3), these theories are undoubtedly conceived and presented as involving a continuum. After all, that was the rationale for the core task, on the traditional approach to renormalization in Sections 2.3 and 2.4: to assign bare coupling constants at each value of the cut-off $d$, so as to recover the physical coupling constants (cf. eq. 2.9). Similarly for $L$ and $\mu$. In a theory assuming a spacetime continuum, there is no a priori obstacle to arbitrarily shortdistance, high-energy phenomena - $L$ can be arbitrarily small, $\mu$ arbitrarily high.

But in condensed matter, the inter-atomic or inter-molecular distance gives a natural lower limit to the length-scales at which we trust our analyses. Thus recall that in Section 3.3.1, I said the critical point involves scale-invariance, 'until we reach molecular dimensions, where, of course, the alternation between the phases breaks down'. More generally: we of course expect our definition of the renormalization group flow, by which one "vector" of physical coupling constants (or: one Hamiltonian or Lagrangian) replaces another as the energy scale increases (distance scale decreases) to break down at molecular or atomic scales. (Or of course at larger length-scales, if some new physics "kicks in" there, which our definition of the flow has disregarded: an example would be spatially periodic inhomogeneities with, say, a mesoscopic period.) Similarly of course, for theoretical models rather than real samples. The classic example is modelling a crystal as a spatially periodic lattice of sites representing atoms or nuclei. In such a model, the lattice spacing provides the natural lower limit. To sum up: at this limit, the renormalization group transformation - the step along the flow towards the ultra-violet that "zooms in" by an increment - cannot be iterated.

\footnotetext{
${ }^{20}$ Cf. e.g. Kadanoff (2013, Section 8.5, p.181). I say there is 'not even a puzzle'; but it can be confusing - witness the exchanges between Cao and Fisher which pepper Cao (1999a).
} 
Mention of lattice models in condensed matter prompts a final remark, to complete my description of quantum field theory. Namely, lattice models are also crucial there: but with a lattice of sites throughout a region of spacetime, rather than space. We can easily state two broad reasons why.

(1): Since quantum field theories are complicated, one often cannot solve one's problem analytically (even with perturbation theory, power series etc.) and so needs to solve it numerically, i.e. on a computer: which involves discretizing the field variables, i.e. assigning them to sites of a lattice rather than the continuum of spacetime points.

(2): I enthused about QCD's asymptotic freedom: that the theory becomes very simple, indeed free, at arbitrarily high energies. But consider the other side of this coin: as the energy is lowered, the interaction becomes stronger. Of course it was realized already in the 1930s that the interaction holds the nucleus together, overcoming the electrostatic repulsion among the protons. Hence its name, 'strong', given long before QCD and asymptotic freedom were found. This strength means a large physical coupling constant, and so difficulties for a perturbative expansion (cf. Section 2.2.1's discussion of $\varepsilon$ and $\alpha$ ): difficulties that led in the 1950s and 1960s to scepticism that any perturbative quantum field theory could describe the interaction. Again there is jargon: the increasing strength at larger distances is called infra-red slavery, and the ever-tighter binding of quarks as they try to separate (which prevents us seeing a lone quark) is called confinement; (e.g. Wilczek 2005, Sections 1.1, 3.1). But jargon aside, the predicament that the theory is complicated and very hard to calculate in prompts lattice models and the hunt for numerical solutions.

\section{Nagelian reflections}

So much by way of reviewing renormalization, in both the traditional and modern approaches. In this final Section, I turn briefly to philosophy. I will confine myself to urging a moral which honours Ernest Nagel. Namely: the material in Sections 2 and 3 fits well with Nagel's account of reduction; and indeed, Hempel's deductivenomological account of explanation. In particular, the idea of universality (Sections 3.1.4 and 3.3.2) is a case of the philosophical idea of multiple realizability, and does not cause problems for Nagelian reduction.

That is good news for me, since in previous work I joined others in endorsing Nagel's account, and in arguing that multiple realizability is no threat to Nagelian reductions (2011, Sections 3 and 4; and for renormalization, 2014). I will not repeat any of the details of those arguments here. ${ }^{21}$ I will just sketch Nagel's account (Section

\footnotetext{
${ }^{21}$ Not that Nagel's doctrines about explanation, reduction and inter-theoretic relations need $m y$ endorsement: especially since, happily, his stock-price is rising. For example, one recent defence of Nagel's account, and similar accounts such as Schaffner's (1967, 1976), is Dizadji-Bahmani, Frigg and Hartmann (2010); and Schaffner has recently given a masterly review of this literature, and defence of his own account (2013). As to multiple realizability not threatening Nagelian reduction,
} 
4.1) and describe how the material I have reviewed illustrates it (Section 4.2). Finally in Section 4.3 I will urge that universality is just multiple realizability.

But before setting out, I note as an appetizer for another occasion, two philosophical topics prompted by the material in Sections 2 and 3.

(1): Real infinities?: Should we acquiesce in the traditional approach's acceptance of infinite bare coupling constants (Section 2.4)? I noted there that most physicists find such physically real, albeit unmeasurable, infinities intellectually uncomfortable; and for all the achievements of the modern approach (especially Sections 3.1, 3.3.2), this approach does not expunge these infinities. So what should a philosopher say about such infinities: are they acceptable? ${ }^{22}$

(2): Instrumentalism?: The dwindling of non-renormalizable terms in the infrared prompted the effective field theory programme, with its vision of a succession of theories, each accurately describing one energy range, but inaccurate beyond itsuggesting an instrumentalist attitude (Section 3.1.3). But of course, this dwindling (and allied technical considerations) do not make such an attitude compulsory, especially if we think there are good prospects for getting an empirically adequate and rigorously defined interacting quantum field theory: prospects that are certainly enhanced by QCD's asymptotic freedom (cf. the end of Section 3.2). So what attitude should we adopt? The answer is bound to be controversial, since it is an example of the broad, and maybe unending, philosophical debate between varieties of instrumentalism and of realism. ${ }^{23}$

\subsection{Nagel endorsed}

The traditional philosophical jargon is that one theory $T_{1}$ reduces to, or is reducible to, another $T_{2}$ if, roughly speaking, $T_{1}$ is a part of $T_{2}$. To explicate this, ${ }^{24} \mathrm{I}$ first introduce mnemonic subscripts, to avoid confusion between $T_{1}$ and $T_{2}$.

For the reducing theory, I will use $b$ : 'b' is for bottom/basic/best; 'bottom' and 'basic' connoting microscopic and fundamental, and 'best' connoting a successor theory. And for the reduced theory, I will use $t$ : ' $\mathrm{t}$ ' is for top/tangible/tainted; 'top' and 'tangi-

I endorse Sober (1999).

${ }^{22}$ In some of his work, Batterman has advocated physically real infinities (e.g. 2005, pp. 235236). But beware: this is a different topic. For it rests on different considerations than infinite bare coupling constants in renormalization theory: viz. (i) singular limits, and (ii) the need to take a limit, so as to describe or explain a distinctive phenomenon, e.g. the thermodynamic limit so as to get a phase transition. For my own assessment of his arguments about (i) and (ii), cf. my (2011a, Section 3; 2014, Section 5.1).

${ }^{23}$ For more discussion, with an emphasis on renormalization and the idea of "reductionism", cf.: (i) Cao (1993), Schweber (1993), Cao and Schweber (1993), Cao (1997, Section 11.4 pp. 339-352); (ii) the brief contrasting discussions by Weinberg (1999, pp. 246-250) and Redhead (1999, pp. 38-40); (iii) responses to Cao and Schweber by Hartmann (2001) and Castellani (2002); and more recently, (iv) Bain (2013, 2013a) and my (2014, Section 5.3) .

${ }^{24}$ Of course, we can and should admit that 'reduction', used as a relation between theories, is vague (my 2011, Section 1.1.2). But this is undoubtedly the core idea. 
ble' connoting macroscopic and observable, and 'tainted' connoting a predecessor theory. Thus I will say that a theory $T_{b} \equiv T_{\text {bottom/basic/best }}$ reduces $T_{t} \equiv T_{\text {top } / \text { tangible/tainted; }}$ and that $T_{t}$ reduces to, or is reducible to, $T_{b}$.

Nagel's main idea is to take theories as linguistic entities, viz. sets of sentences closed under deducibility, and hold that $T_{t}$ is reduced to $T_{b}$ by:

(1) Deducibility: being shown to be logically deducible from $T_{b}$, usually together with some judiciously chosen definitions; and

(2) Constraints: the deduction satisfying some informal constraints, e.g. about the derivation counting an explanation of its conclusion.

Before I give some details about (1) and (2), in Sections 4.1.1 and 4.1.2 respectively, my first and most important comment is that taking theories as sets of sentences does not imply that the language of the theories, or the notion of deducibility, be formalized (let alone first-order). Nagel and his supporters (including Schaffner et al. listed in footnote 21) of course know that scientific theories are not formalized, and are hardly likely to be, even in mathematized subjects like physics. But that does not prevent one undertaking to assess whether there are relations of reduction between theories (in Nagel's sense; or indeed, in another one). The informality merely makes one's specification of the theories, definitions and deducibility relation, somewhat vague; and so one's ensuing assessments are correspondingly tentative. ${ }^{25}$

\subsubsection{Deducibility}

The syntactic conception of theories immediately gives a notion of $T_{t}$ being a part of $T_{b}$, viz. when $T_{t}$ 's sentences, i.e. theorems, are a subset of $T_{b}$ 's.

In logic, with its formalized languages, this is called $T_{t}$ being a sub-theory of $T_{b}$. But the notion of course applies to unformalized languages. One just has to recognize that which sentences are in a given theory is almost always vague, even when the theory's name is very specific (e.g. 'equilibrium classical statistical mechanics of $N$ point-particles in a cube with side-length $l$ ', rather than 'classical statistical mechanics'). And to avoid merely verbal disputes, one must adopt a swings-androundabout attitude about whether a given $T_{t}$ is part of a given $T_{b}$. For it all depends on how one makes the two theories, i.e. their sets of sentences, precise; (cf. my 2011, Section 3.1.2; and Schaffner (2013, Section II p. 7, Section VI, p. 29)).

So although scientific theories are of course unformalized, I will continue to use logical jargon, saying e.g. that a theory has a set of sentences, a language has a set of predicates etc. I will also set aside the questions of what a theory's underlying

\footnotetext{
${ }^{25}$ I agree that the tenor of Nagel's writings - and of his times - also suggests a narrower, and I admit implausible, account requiring that theories, definitions and deductions, be formalized. (Thanks to Bob Batterman and Jim Weatherall for emphasizing this.) I say a bit more about this, and the rival "semantic view" of theories in my (2011, Sections 3, 4; 2014, Section 1.2). I should also note that, on the other hand, some writers have for various reasons criticized the notion of a scientific theory, even if theories are not taken as linguistic entities; and have even invoked quantum field theories in their support. I reply to this line of thought, in the companion paper (2014, Section 4.1).
} 
logic is (first-order vs. second-order etc.) and what its mathematical apparatus is (containing set theory, calculus etc.), since for unformalized theories these questions also are vague. So I just refer non-commitally to 'deduction'.

However, one still needs to avoid confusion arising from the same predicate, or other non-logical symbol, occurring in both theories, but with different intended interpretations. This can be addressed by taking the theories to have disjoint non-logical vocabularies, and then defining $T_{t}$ to be a definitional extension of $T_{b}$, iff one can add to $T_{b}$ a set $D$ of definitions, one for each of $T_{t}$ 's non-logical symbols, in such a way that $T_{t}$ becomes a sub-theory of the augmented theory $T_{b} \cup D$. That is: In the augmented theory, we can deduce every theorem of $T_{t}$.

Note that here again, I mean the word 'definition' as it is used in logic: roughly speaking, a definition states that a given "new" non-logical symbol e.g. a predicate, the definiens, is co-extensive with some (maybe very long) open sentence, the definiendum. There is no formal requirement that a definition be faithful to some previous meaning (or some other properties) of the definiens: I shall return to this in Section 4.1.2.A.

This is the core formal part of Nagelian reduction: (1) Deducibility, in the above notation. The definitions are usually called 'bridge laws' (or 'bridge principles' or 'correspondence rules'). ${ }^{26}$

\subsubsection{Informal constraints on reduction}

4.1.2.A: Deducibility is too weak Various philosophers have said that even if $T_{t}$ is a definitional extension of $T_{b}$, there can be non-formal aspects of $T_{t}$ that are not encompassed by (are not part of) the corresponding aspects of $T_{b}$; and that, at least in some cases, these aspects seem essential to $T_{t}$ 's functioning as a scientific theory. Nagel agrees with the general point that deducibility is too weak, and (1961, pp. 358-363) adds some informal conditions, mainly motivated by the idea that the reducing theory $T_{b}$ should explain the reduced theory $T_{t}$; and following Hempel, he conceives explanation in deductive-nomological terms. Thus he says, in effect, that $T_{b}$ reduces $T_{t}$ iff:

(i): $T_{t}$ is a definitional extension of $T_{b}$; and

(ii): in each of the definitions of $T_{t}$ 's terms, the definiens in the language of $T_{b}$ must play a role in $T_{b}$; so it cannot be, for example, a heterogeneous disjunction.

Nagel's proposal (ii) has got some good press; e.g. Sklar (1967, pp. 119-123), Nickles (1973, pp. 190-194). But some philosophers neglect this proposal and-or object that it does not secure what is needed. The most common objection appeals to multiple realizability. The leading idea is that the definiens of a multiply realizable

\footnotetext{
${ }^{26}$ The standard references are Nagel (1961, pp. 351-363; and 1979); cf. also Hempel (1966, Chapter 8). Discussions of bridge laws are at Nagel (1961, pp. 354-358; 1979, 366-368) and Hempel (1966, pp. 72-75, 102-105). My notation Deducibility thus covers the conditions Nagel calls 'derivability' (about logical consequence) and 'connectability' (about definitions); cf. the discussion in Schaffner (2013, Section I). In Section 4.1.2 I will mention revisions by authors such as Schaffner.
} 
property shows it to be too "disjunctive" to be suitable for scientific explanation, or to enter into laws. And pace Nagel's proposal (ii) above, some philosophers think that multiple realizability prompts a non-Nagelian account of reduction.

These lines of thought are most familiar in the philosophy of mind, with the multiple realizability argument of Putnam (1975) and Fodor (1974). Kim's work provides an example which specifically targets Nagelian reduction. He calls it 'philosophically empty' and 'irrelevant', because Nagelian bridge laws are 'brute unexplained primitives'; (1999, p. 134; 2006, p. 552; and similarly in other works e.g. (2005, pp. 99-100): cf. also e.g. Causey (1972, p. 412-421)). Kim's own account, called a 'functional model of reduction', takes reduction to include:

(a) functional definitions of the higher-level properties $P$ etc., i.e. definitions that quantify over properties so as to characterise $P$ by its pattern of relations (usually causal and-or nomic) to various other properties; and

(b) a lower-level description of the (variety-specific) realizers of $P$ etc., and of how they fulfill the functional roles spelt out in (a).

But as announced, I reject these lines of thought. I believe multiple realizability, and functional definitions, give no argument against definitional extension; nor even against stronger notions of reduction like Nagel's, that add further constraints additional to deducibility, e.g. about explanation. That is: I believe that such constraints are entirely compatible with multiple realizability. I maintain (2011, Sections 3.1.1 and 4.1.1) that this was shown persuasively by Sober (1999) ${ }^{27}$. I will not repeat the rebuttal. Let me just sum up: there is no tension between Nagelian reduction and the fact of multiple realizability.

4.1.2.B: Deducibility is too strong The other traditional criticism of Nagelian reduction goes in the "opposite direction". Instead of saying that Deducibility is too weak, it says that Deducibility is too strong. The idea is that in many cases where $T_{b}$ reduces $T_{t}, T_{b}$ corrects, rather than implies, $T_{t}{ }^{28}$

But as an objection to Nagel, this is an ignoratio elenchi. For Nagel himself made this point. He said that a case in which $T_{t}$ 's laws are a close approximation to what strictly follows from $T_{b}$ should count as reduction. He called this approximative

\footnotetext{
${ }^{27}$ Sober mostly targets Putnam and Fodor; for critiques of Kim, cf. Marras (2002, pp. 235-237, 240-247), Needham (2009, pp. 104-108)

${ }^{28}$ This objection is made by e.g. Kemeny and Oppenheim (1956), and Feyerabend (1962). One standard example is Newtonian gravitation theory $\left(T_{b}\right)$ and Galileo's law of free fall $\left(T_{t}\right)$. This $T_{t}$ says that bodies near the earth fall with constant acceleration. This $T_{b}$ says that as they fall, their acceleration increases, albeit by a tiny amount. But surely $T_{b}$ reduces $T_{t}$. And similarly in many famous and familiar examples of reduction in physics: wave optics corrects geometric optics, relativistic mechanics corrects Newtonian mechanics etc.

These examples put limiting relations between theories centre-stage. And quite apart from assessing Nagel, such relations have long been a major focus for philosophers of the physical sciences: as also in recent years, cf. e.g. Batterman (2002, 2013), Norton (2012). My own views about these relations are in (2011a): which takes as one of its examples, phase transitions, especially Section 3.3.1's topic of critical points; (cf. also Bouatta and Butterfield (2011, 2012).
} 
reduction (1979, pp. 361-363, 371-373). (Cf. also Hempel (1965, p. 344-346; 1966, pp. 75-77). In a similar vein, Schaffner (1967, p. 144; 1976, p. 618) requires a strong analogy between $T_{t}$ and its corrected version, i.e. what strictly follows from $T_{b}$ ).

Against this, critics have complained that it is too programmatic, since it gives no general account of when an approximation or analogy is close enough. But in Nagel's and Schaffner's defence, I would say that (a) we should not expect, and (b) we do not need, any such general account. ${ }^{29}$ What matters, both scientifically and conceptually, is that in a given case we can deduce that $T_{t}$ 's proposition (whether a description of particular fact, or a general theorem/law) is approximately true; and that we can quantify how good the approximation is.

\subsection{Renormalizability deduced at low energies as a family of Nagelian reductions}

I now conclude by discussing how renormalization illustrates Nagelian reduction. I will confine myself to the topic announced in Section 1: how the modern approach, with its explanation that our good fortune in having renormalizable theories is generic (Section 3.1), accords with Nagelian reduction.

As I said in Section 1, I think philosophers should take note, not just of this specific achievement, but of the general idea of a space of theories. This fosters a novel and more ambitious kind of explanatory project than the familiar ones of explaining an individual event, or a single law, or (as in Section 4.1) a theory as a part of, or a good approximation to, another. Namely: to explain a feature of a whole class of theories in a unified way in terms of the structure of the space of theories.

But I will not develop this general theme, but instead concentrate on my main claim, as follows:

The deduction from a given theory $T_{b}$ that describes (perhaps using nonrenormalizable terms) high-energy physics, of a renormalizable theory $T_{t}$ describing low energy physics, is a Nagelian reduction. Besides: for different pairs of theories $T_{b}$ and $T_{t}$, varying across the space of quantum field theories, the reductive relation is similar, thanks to a shared definition of the renormalization group flow, i.e. of the renormalization scheme.

I will fill this out with five short remarks, rehearsing previous material. As regards the philosophical assessment of Nagel's account of reduction, the most important of these remarks are (3), about approximative reduction, and (4), about unity among a family of reductions.

\footnotetext{
${ }^{29}$ I am not alone in this defence: cf. Nickles (1973, p. 189, 195), Schaffner (2013, Section III) and Dizadji-Bahmani, Frigg and Hartmann (2010: Section 3.1, p. 409f.). In a similar vein, the latter argue (their Section 5) that Nagelian reduction does not need to settle once for all whether bridge laws state "mere" correlations, law-like correlations or property-identities: (which are Nagel (1961)'s three options, at pp. 354-357). I entirely agree, pace authors like Kim and Causey cited in Section 4.1.2.A.
} 
(1): I have specified a theory by a Hamiltonian or Lagrangian. Recall the vector of physical coupling constants $g_{1}(\mu), \ldots, g_{N}(\mu)$ which I first introduced in Section 2.5.1, and took as a point in a space of theories at the start of Section 3.

(2): A renormalization scheme that defines a flow towards lower energies (a scheme for coarse-graining so as to eliminate higher-energy degrees of freedom) amounts to the set of definitions $D$, in logicians' broad sense (Section 4.1.1), needed to make the deduction of $T_{t}$ from $T_{b}$ go through.

(3): Since at low energies any non-renormalizable terms in $T_{b}$ still make non-zero, albeit dwindling, contributions to the theory's predictions (probabilities for various processes), we have here an approximative reduction (Section 4.1.2.B); though the approximation gets better and better as the energy decreases.

(4): A given renormalization scheme (definition of the flow) works to show that many theories $T_{b}$ lead to a renormalizable low-energy theory $T_{t}$. This unity is striking. Hence this Section's title's use of the word 'family': since 'family' connotes resemblance, which 'set' does not.

(5): Agreed: no single renormalization scheme works to prove that all possible theories have dwindling non-renormalizable contributions in the infra-red. And as I have often admitted: the proofs concerned are often not mathematically rigorous. But the various renormalization schemes that have been devised do not contradict one another, and in fact mesh in various ways. So it is fair to say that a generic quantum field theory has dwindling non-renormalizable contributions in the infra-red. As I said in Section 3.1: a satisfying explanation of our good fortune. ${ }^{30}$

So to sum up: the modern approach to renormalization gives a stunning case of explaining something that is otherwise - i.e. on the traditional approach - a coincidence. The coincidence is, in short, that the theory in question, e.g. quantum electrodynamics, has a feature, viz. renormalizability, that seems crucial to it "making sense". This feature also holds of other theories; indeed, it holds of the whole standard model of particle physics, which combines quantum electrodynamics with quantum theories of the weak and strong forces. Thus the coincidence is so grand and important that it seems like manna from heaven; or more prosaically, it seems that renormalizability is in some sense an a priori selection-principle for quantum theories. But adopting the modern approach, we can deduce that what seemed manna from heaven is in a sense to be expected.

\subsection{Universality is multiple realizability}

My final point is a brief ancillary one. In Section 3.1.4, I introduced 'universality' as jargon for the idea that dissimilar theories might have similar infra-red behaviour: in particular, the same infra-red fixed point. In Section 3.3.2, we saw vivid examples of

\footnotetext{
${ }^{30}$ That a satisfying explanation should show the explanandum to be generic has of course been a theme in cosmological speculations about explaining initial conditions: not just nowadays, but over the centuries; cf. McMullin (1993).
} 
this. The dissimilar theories were of utterly different quantities in utterly different systems: e.g. a density difference in a mixture of water and steam, or in a mixture of two phases of liquid helium; or the magnetization of a piece of iron or nickel. And the similar behaviour was quantitatively exact, albeit arcane: the same values of critical exponents in power laws describing near-critical behaviour. In the examples just mentioned, it was the critical exponent $\beta$ in eq. 3.4. Corresponding remarks apply to the exponents $\gamma, \eta$ and $\nu$, in eq. 3.5, 3.6 and 3.8 respectively.

These examples obviously illustrate multiple realizability in the sense introduced in Section 4.1.2.A. To take just my first example: having a value of $\beta$ equal to about 0.35 as in eq. 3.4 is a multiply realizable property. A water-steam mixture has it; so does a mixture of two phases of liquid helium. Similarly for Section 4.1.2.A's idea of a functional definition of a property, viz. specifying a pattern of relations to other properties: clearly, having $\beta \approx 0.35$ is a functional property.

But recall that I join Sober (1999) in seeing no tension between Nagelian reduction and multiple realizability, with the consequent need for functional definitions. So I also see no trouble for Nagelian reduction in universality: e.g. in critical exponents, whether calculated using the renormalization group or using older approaches like mean field theory (cf. footnote 19). In particular, I see no trouble for Section 4.2's main claim that to coarse-grain a class of theories towards their common infra-red fixed point - zooming out our descriptions in each theory, towards longer distancesis to give a unified family of Nagelian reductions.

Acknowledgements:- JB thanks: Sebastien Rivat, Nic Teh, and seminar audiences in Cambridge for discussion and comments on early versions (2012); Jonathan Bain, Bob Batterman, Tian Yu Cao, Elena Castellani, and Jim Weatherall for generous comments on a later draft (2013); and audiences at Columbia University, New York and Munich (2013). Thanks also to the editors and a very perceptive referee. While I have incorporated most of these comments, I regret that I could not act on them all. I thank the Journal of Philosophy for allowing some overlap (in Sections 3.1.2 - 3.1.4 and 4.2) with the companion paper (2014). This work was supported in part by a grant from the Templeton World Charity Foundation, whose support is gratefully acknowledged.

\section{References}

Aitchison, I. (1985), 'Nothing's plenty: the vacuum in quantum field theory', Contemporary Physics 26, p. 333-391.

Applequist, T. and Carazzone, J. (1975), 'Infra-red singularities and massive fields', Physical Review D11, pp. 2856-2861.

Baez, J. (2006), 'Renormalizability': (14 Nov 2006):

http://math.ucr.edu/home/baez/renormalizability.html 
Baez, J. (2009), 'Renormalization made easy': (5 Dec 2009): http://math.ucr.edu/home/baez/renormalization.html

Bain, J. (2013), 'Effective Field Theories', in Batterman, R. (ed.) The Oxford Handbook of Philosophy of Physics, Oxford University Press, pp. 224-254.

Bain, J. (2013a), 'Emergence in Effective Field Theories', European Journal for Philosophy of Science, 3, pp. 257-273. (DOI 10.1007/s13194-013-0067-0)

Batterman, R. (2002), The Devil in the Details, Oxford University Press.

Batterman, R. (2005), 'Critical phenomena and breaking drops: infinite idealizations in physics', Studies in History and Philosophy of Modern Physics 36B, pp. 225-244.

Batterman, R. (2010), 'Reduction and Renormalization', in A. Huttemann and G. Ernst, eds. Time, Chance, and Reduction: Philosophical Aspects of Statistical Mechanics, Cambridge University Press, pp. 159-179.

Batterman, R. (2013), 'The tyranny of scales', in The Oxford Handbook of the Philosophy of Physics, ed. R. Batterman, Oxford University Press, pp. 255-286.

Binney, J., Dowrick, N, Fisher, A. and Newman, M. (1992), The Theory of Critical Phenomena: an introduction to the renormalization group, Oxford University Press.

Bouatta, N. and Butterfield, J. (2011), 'Emergence and Reduction Combined in Phase Transitions', in J. Kouneiher, C. Barbachoux and D.Vey (eds.), Proceedings of Frontiers of Fundamental Physics 11 (American Institute of Physics); pp.?? . Available at: http:// philsci-archive.pitt.edu/8554/ and at: http://arxiv.org/abs/1104.1371

Bouatta, N. and Butterfield, J. (2012), 'On emergence in gauge theories at the 't Hooft limit', forthcoming in European Journal for Philosophy of Science, http://philsciarchive.pitt.edu/9288/

Brown, L. ed. (1993), Renormalization: from Lorentz to Landau (and Beyond), Springer.

Butterfield, J. (2004), 'Some Aspects of Modality in Analytical Mechanics', in P. Weingartner and M. Stoeltzner (eds), Formale Teleologie und Kausalitat in der Physik, Mentis 2004; pp. 160-198;

physics/0210081; and http://philsci-archive.pitt.edu/archive/00001192/

Butterfield, J. (2011), 'Emergence, Reduction and Supervenience: a Varied Landscape', Foundations of Physics, 41, pp. 920-960. At Springerlink: doi:10.1007/s10701011-9549-0; http://arxiv.org/abs/1106.0704: and at: http://philsci-archive.pitt.edu/5549/

Butterfield, J. (2011a), 'Less is Different: Emergence and Reduction Reconciled', in Foundations of Physics 41, pp. 1065-1135. At: Springerlink (DOI 10.1007/s10701010-9516-1); http://arxiv.org/abs/1106.0702; and at: http://philsci-archive.pitt.edu/8355/

Butterfield, J. (2014), 'Reduction, emergence and renormalization', The Journal of Philosophy 111, pp. 5-49.

Cao, T.Y. (1993), 'New philosophy of renormalization: from the renormalization group to effective field theories', in Brown ed. (1993), pp. 87-133.

Cao, T.Y. (1997), Conceptual Developments of Twentieth Century Field Theories, Cambridge University Press. 
Cao, T.Y., ed. (1999), Conceptual Foundations of Quantum Field Theory, Cambridge University Press.

Cao, T.Y. (1999a), 'Renormalization group: an interesting yet puzzling idea', in Cao (ed.) (1999), pp. 268-286.

Cao, T.Y and Schweber, S. (1993), 'The conceptual foundations and the philosophical aspects of renormalization theory, Synthese 97, pp. 33-108.

Castellani, E. (2002), 'Reductionism, emergence and effective field theories', Studies in the History and Philosophy of Modern Physics 33, 251-267.

Causey, R. (1972), 'Attribute-identities and micro-reductions', Journal of Philosophy 67, pp. 407-422.

Dizadji-Bahmani, F., Frigg R. and Hartmann S. (2010), 'Who's afraid of Nagelian reduction?', Erkenntnis 73, pp. 393-412.

Feyerabend, P. (1962), 'Explanation, reduction and empiricism', in H. Feigl and G. Maxwell (eds.) Minnesota Studies in Philosophy of Science 3, University of Minnesota Press, pp. 28-97.

Feynman, R. (1985), QED, Princeton University Press.

Fodor, J. (1974), 'Special Sciences (Or: the disunity of science as a working hypothesis), Synthese 28, pp. 97-115.

Giere, R. (1995), Science Without Laws, University of Chicago Press.

Gross, D. (1999), 'Renormalization groups' in P. Deligne et al. (eds.), Quantum Fields and Strings; a Course for Mathematicians, American Mathematical Society; pp. 551-596.

Hartmann, S. (2001). 'Effective field theories, reductionism and scientific explanation', Studies in History and Philosophy of Modern Physics 32, pp. 267-304.

Hempel, C. (1965), Aspects of Scientific Explanation, New York; the Free Press.

Hempel, C. (1966), Philosophy of Natural Science, Prentice-Hall.

Hesse, M. (1965), Forces and Fields, ?Macmillan.

Horejsi, J. (1996), 'Electroweak interactions and high-energy limit', Czechoslovak Journal of Physics 47 (1997), pp. 951-977; http://arxiv.org/abs/hep-ph/9603321

Jaffe, A. (1999), 'Where does quantum field theory fit into the big picture?', in Cao (ed.) (1999), pp. 136-146.

Jaffe, A. (2008), 'Quantum theory and relativity', in Contemporary Mathematics (Group Representations, Ergodic Theory, and Mathematical Physics: A Tribute to George W. Mackey), R. Doran, C.Moore, and R. Zimmer, (eds.), 449, pp. 209246; available at http://arthurjaffe.org

Kadanoff, L. (2009), 'More is the same: mean field theory and phase transitions', Journal of Statistical Physics 137,pp. 777-797.

Kadanoff, L. (2013), 'Theories of matter: infinities and renormalization', in The Oxford Handbook of the Philosophy of Physics, ed. R. Batterman, Oxford University Press, pp. 141-188.

Kemeny, J and Oppenheim P. (1956), 'On reduction', Philosophical Studies 7, pp. $6-19$. 
Kim, J. (1999), 'Making sense of emergence', Philosophical Studies 95, pp. 3-36; reprinted in Bedau and Humphreys (2008); page reference to the reprint.

Kim, J. (2005), Physicalism, or Something Near Enough, Princeton University Press.

Kim, J. (2006), 'Emergence: Core Ideas and Issues', Synthese 151, pp. 547-559.

Kogut, J. and Stephanov, M. (2004), The Phases of Quantum Chromodynamics, Cambridge University Press.

Lange, M. (2002), An Introduction to the Philosophy of Physics, Blackwell.

Lautrup, B. and Zinkernagel, H. (1999), 'g-2 and the trust in experimental results', Studies in the History and Philosophy of Modern Physics 30, pp. 85-110.

McMullin, E. (1993), 'Indifference principle and anthropic principle in cosmology', Studies in the History and Philosophy of Science 24, pp. 359-389.

McMullin, E. (2002), 'Origins of the field concept in physics', Physics in Perspective

Marras, A. (2002), 'Kim on reduction', Erkenntnis 57, pp. 231-257.

Menon, T. and Callender, C. (2013), 'Turn and face the ch-ch-changes: philosophical questions raised by phase transitions', in The Oxford Handbook of the Philosophy of Physics, ed. R. Batterman, Oxford University Press, pp. 189-223.

Moffat, J. (2010), 'Ultraviolet Complete Electroweak Model Without a Higgs Particle', European Physics Journal Plus 126 (2011), p. 53; http://arxiv.org/pdf/1006.1859v5

Morrison, M. (2012), 'Emergent physics and micro-ontology', Philosophy of Science 79, pp. 141-166.

Nagel, E. (1961), The Structure of Science: Problems in the Logic of Scientific Explanation, Harcourt.

Nagel, E. (1979), 'Issues in the logic of reductive explanations', in his Teleology Revisited and other essays in the Philosophy and History of Science, Columbia University Press; reprinted in Bedau and Humphreys (2008); page reference to the reprint.

Needham, P. (2009), 'Reduction and emergence: a critique of Kim', Philosophical Studies 146, pp. 93-116.

Nickles, T. (1973), 'Two concepts of inter-theoretic reduction', Journal of Philosophy 70, pp. 181-201.

Norton, J. (2012), 'Approximation and idealization: why the difference matters', Philosophy of Science 74, pp. 207-232.

Putnam, H. (1975), 'Philosophy and our mental life', in his collection Mind, Language and Reality, Cambridge University Press, pp. 291-303.

Redhead, M. (1999), 'Quantum field theory and the philosopher, in Cao (ed.) (1999), pp. 34-40.

Schaffner, K. (1967), 'Approaches to reduction', Philosophy of Science 34, pp. 137-147.

Schaffner, K. (1976), 'Reductionism in biology: problems and prospects' in R. Cohen et al. (eds), PSA 1974, pp. 613-632. 
Schaffner, K. (2013), 'Ernest Nagel and reduction', Journal of Philosophy 109, pp. 534-565.

Schweber, S. (1993), 'Changing conceptualization of renormalization theory', in Brown ed. (1993), pp. 135-166.

Schweber, S. (1994), QED and the Men who Made It, Princeton University Press.

Sklar, L. (1967), 'Types of intertheoretic reduction', British Journal for the Philosophy of Science 18, pp. 109-124.

Sober, E. (1999), 'The multiple realizability argument against reductionism', Philosophy of Science 66, pp. 542-564.

Symanzik, K. (1973), 'Infra-red singularities and small-distance behaviour analysis', Communications in Mathematical Physics 34, pp. 7-36.

Teller, P. (1989), 'Infinite renormalization', Philosophy of Science 56, pp. 238-257; reprinted with minor corrections as Chapter 7 of his An Interpretive Introduction to Quantum Field Theory (1995), Princeton University Press.

Weinberg, S. (1995), The Quantum Theory of Fields, volume 1, Cambridge University Press.

Weinberg, S. (1995a), The Quantum Theory of Fields, volume 2, Cambridge University Press.

Weinberg, S. (1999), 'What is quantum field theory and what did we think it was', in Cao ed. (1999), pp. 241-251. Also at: arxiv: hep-th/9702027

Wightman, A. (1999), 'The usefulness of a general theory of quantized fields', in Cao (ed.) (1999), pp. 41-46.

Wilczek, F. (2005), 'Asymptotic freedom: from paradox to paradigm', (Nobel Prize Lecture 2004), Proceedings of the National Academy of Science, 102, pp. 84038413; available at: hep-ph/0502113

Wilson, K. (1979), 'Problems in Physics with Many Scales of Length', Scientific American 241, pp. 158-179.

Zuchowski (2013), 'For electrodynamic consistency', forthcoming in Studies in the History and Philosophy of Modern Physics. 